\title{
p-TORSION POINTS ON ELLIPTIC CURVES DEFINED OVER QUADRATIC FIELDS
}

\author{
FUMIYUKI MOMOSE*
}

Let $p$ be a prime number and $k$ an algebraic number field of finite degree $d$. Manin [14] showed that there exists an integer $n=n(k, p)(\geqq 0)$ which satisfies the condition

$$
E(k)_{p^{\infty}} \subseteq \operatorname{ker}\left(p^{n}: E \longrightarrow E\right)
$$

for all elliptic curves $E$ defined over $k$. Here, $E_{p^{\infty}}=\bigcup_{m \geqq 1} E_{p^{m}}$ and $E_{p^{m}}=\operatorname{ker}\left(p^{m}: E \rightarrow E\right)$. We denote by $n=n(k, p)$ the least non-negative integer satisfying the above condition. For $k=\boldsymbol{Q}$, we know that $n(\boldsymbol{Q}, 2)=$ $3, n(\boldsymbol{Q}, 3)=2, n(\boldsymbol{Q}, 5)=n(\boldsymbol{Q}, 7)=1$ and $n(\boldsymbol{Q}, p)=0$ for $p \geqq 11$ (cf. [10], $[16,17],[20],[22])$. For quadratic fields $k$, Kenku [6, 8, 9] showed that $n(k, 2) \leqq 4, n(k, 3)=2, n(k, 5)=n(k, 7)=1, n(k, 17)=n(k, 19)=n(k, 23)=0$ and $n(k, p)=0$ for the primes $p ; p \geqq 181, p \neq 191$ and $\# J_{0}^{-}(p)(Q)<\infty$. Here $J_{0}(p)$ is the jacobian variety of the modular curve $X_{0}(p), w_{p}$ is the automorphism of $J_{0}(p)$ induced by the fundamental involution $w_{p}:(E, A) \mapsto$ $\left(E / A, E_{p} / A\right)$ of $X_{0}(p)$ and $J_{0}^{-}(p)=J_{0}(p) /\left(1+w_{p}\right) J_{0}(p)$ (see [17]). Our result for quadratic fields $k$ is the following.

Theorem A. Let $k$ be any quadratic field and $n=n(k, p)$ as above. Then

$$
\begin{aligned}
& n(k, 11) \leqq 1 \\
& n(k, 13) \leqq 1
\end{aligned}
$$

and $n(k, p)=0$ for the primes $p \geqq 17$ satisfying the condition $\# J_{0}^{-}(p)(\boldsymbol{Q})<\infty$.

For $p=2,11$ and $13, n(k, p)$ depends on $k$ (see (3.3)). For the primes $p, 17 \leqq p<300$, except for $p=151,199,227$ and 277 , the condition $\# J_{0}^{-}(p)(Q)<\infty$ is satisfied ([17] p. 40, [35] Table 5 pp. 135-141). We conjecture $n(k, p)=0$ for $p \geqq 17$. Our method used for quadratic fields can

Received December 1, 1983.

* Supported in part by Japan-U.S. exchange fund. 
be applied to some other number fields. For example we get the following.

Theorem B. Let $k$ te any cubic field and $n=n(k, p)$ as above. Then

$$
\begin{aligned}
& n(k, 2) \leqq 5 \\
& n(k, 3)=2 \\
& n(k, 17) \leqq 1
\end{aligned}
$$

and $n(k, p)=0$ for $p=19,23,41,47,59,71$ and the primes $p ; p \geqq 79$, $p \neq 79, p \neq 109$ and $\# J_{0}^{-}(p)(Q)<\infty$.

We here give a sketch of the proof of Theorem A above for the case $p \geqq 23, p \neq 37$. Suppose that there exists a non cuspidal $k$-rational point $x$ on $X_{1}(p)$. Under the condition as in Theorem $\mathrm{A}$, one gets a rational function $g$ on $X_{0}(p)$ defined over $\boldsymbol{Q}$ such that

$$
(g)=(x)+\left(x^{\sigma}\right)+2(\infty)-\left(w_{p}(x)\right)-\left(w_{p}\left(x^{\sigma}\right)\right)-2(0),
$$

where $1 \neq \sigma \in \operatorname{Gal}(k / Q)$ and $0, \infty$ are the cusps on $X_{0}(p)$, Section 2. For $p \geqq 181(p \neq 191)$, Kenku [9] proved that such function $g$ does not exist, using an Ogg's idea [22,24]: The upper semicontinuity gives a non constant rational function $h\left(\mid \boldsymbol{F}_{2}\right)$ on $\mathscr{X}_{0}(p) \otimes \boldsymbol{F}_{2}$ with $(h)_{\infty}<$ an effective divisor of degree 4 , which leads the inequality $\# \mathscr{X}_{0}(p)\left(F_{p}\right) \leqq 10$. For the remaining $p$, we use the following two methods: (1) The condition $\left(w_{p}^{*} g\right)=-(g)(\neq 0)$ shows that $w_{p}^{*}(g)=a / g$ for $a \in \boldsymbol{Q}^{\times}$. Let $y_{i}$ be the fixed points of $w_{p}$ on $X_{0}(p)$ and put $D=\Sigma_{i}\left(y_{i}\right)$. Then one sees that $(g-\sqrt{a})_{0}>\Sigma^{\prime}\left(y_{i}\right)$ and $(g+\sqrt{a})_{0}>\Sigma^{\prime \prime}\left(y_{i}\right)$ with $D=\Sigma^{\prime}\left(y_{i}\right)+\Sigma^{\prime \prime}\left(y_{i}\right)$. This notion and a study on $y_{i}$ give the inequality that the degree of $D \leqq 4$, (2.3). This criterion gives the proof, except for $p=43,67,73,97$ and 163. (2) The upper semicontinuity and a study on the action of $w_{p}$ on $\mathscr{X}_{0}(p) \otimes \boldsymbol{F}_{2}$ give a non constant rational function $h\left(\mid \boldsymbol{F}_{2}\right)$ on $\mathscr{X}_{0}^{+}(p) \otimes \boldsymbol{F}_{2}$ with $(h)_{\infty}<2$ (cusp) (2.4), where $\mathscr{X}_{0}^{+}(p)=\mathscr{X}_{0}(p) /\left\langle w_{p}\right\rangle$. Then $\# \mathscr{X}_{0}^{+}(p)\left(F_{2}\right) \leqq 5$ and $\# \mathscr{X}_{0}^{+}(p)\left(\boldsymbol{F}_{4}\right) \leqq 9$, which complete the remaining case. For $p=13$ and 37 , we apply other methods.

For the case $p<300$, we get an estimate of $n=n(k, p)$ by an integer which depends only on $k$ and $p$ (see $\S 2$ ). We add the table in Section 4.

The author thanks to B. Mazur, T. Sekiguchi and K. Cho for their useful remarks on curves.

Notation. For a prime number $q, \boldsymbol{Q}_{q}^{\text {ur }}$ denotes the maximal unramified extension of $\boldsymbol{Q}_{q}$. Let $K$ be a finite extension of $\boldsymbol{Q}, \boldsymbol{Q}_{q}$ or $\boldsymbol{Q}_{q}^{\text {ur }}$, and $A$ an 
abelian variety defined over $K$. Then $\mathcal{O}_{K}$ denotes the ring of integers of $K, A_{\mathscr{O}_{K}}$ denotes the Néron model of $A$ over the base $\mathcal{O}_{K}$.

\section{§1. Preliminaries}

Let $p$ be a prime number, $X_{1}\left(p^{r}\right)$ (resp. $X_{0}\left(p^{r}\right)$ ) the modular curve (defined over $\boldsymbol{Q}$ ) which corresponds to the modular group $\Gamma_{1}\left(p^{r}\right)$ (resp. $\left.\Gamma_{0}\left(p^{r}\right)\right)$. For $p^{r} \geqq 5, X_{1}\left(p^{r}\right)$ is the coarse moduli space $(/ Q)$ of the isomorphism classes of the generalized elliptic curves $E$ with a torsion point $P$ of order $p^{r}$ up to the isomorphism $(-1)_{E}: E \cong E$. We denote by $Y_{1}\left(p^{r}\right)$, $Y_{0}\left(p^{r}\right)$ the affine open subschemes $X_{1}\left(p^{r}\right) \backslash\{$ cusps $\}$ and $X_{0}\left(p^{r}\right) \backslash\{$ cusps $\}$, respectively. Let $k$ be a number field and $x$ a $k$-rational point on $Y_{1}\left(p^{r}\right)$ (resp. $Y_{0}\left(p^{r}\right)$ ). Then there exists an elliptic curve $E$ defined over $k$ with a torsion point $P$ of order $p^{r}$ (resp. a cyclic subgroup $A$ of rank $p^{r}$ ) defined over $k$ (see [2] VI Proposition (3.2)). Let $f: X_{1}\left(p^{r}\right) \rightarrow X_{0}\left(p^{r}\right)$ be the natural morphism: $(E, \pm p) \rightarrow(E,\langle P\rangle)$, where $\langle P\rangle$ is the cyclic subgroup generated by $P$. Then $f$ is a Galois covering with the Galois group $\bar{\Gamma}\left(p^{r}\right)=$ $\Gamma_{0}\left(p^{r}\right) / \pm \Gamma_{1}\left(p^{r}\right)\left(\simeq\left(Z / p^{r} Z\right)^{\times} / \pm 1\right)$. For an integer $i$ prime to $p,[i](=[-i])$ denotes the element of $\bar{\Gamma}\left(p^{r}\right)$ respresented by $g \in \Gamma_{0}\left(p^{r}\right), g \equiv\left(\begin{array}{ll}i & * \\ 0 & *\end{array}\right) \bmod p^{r}$. The action of [i] is defined by $(E, \pm P) \rightarrow(E, \pm i \cdot P)$. Let $w=w_{p^{r}}$ be the fundamental involution of $X_{0}\left(p^{r}\right):(E, A) \mapsto\left(E / A, E_{p^{r}} / A\right)$ and $X_{0}^{+}\left(p^{r}\right)$ the quotient $X_{0}\left(p^{r}\right) /\langle w\rangle$. For a point on a modular curve, $\rightarrow X_{0}(1)$ (= the projective $j$-line $/ \boldsymbol{Q}), j(x)$ denotes the modular invariant of $x$. We here explain the fixed points of $w_{p}$ on $X_{0}(p)$ and add a table of the MordellWeil groups of subcoverings $X: X_{1}\left(p^{r}\right) \rightarrow X \rightarrow X_{0}\left(p^{r}\right)$. Further we discuss the fixed points of $w_{p}$ on $\mathscr{X}_{0}(p) \otimes Z_{2}$ and prepare some lemmas on curves, which will be used in Section 2 .

(1.1) The ramification points of $Y_{1}\left(p^{r}\right) \longrightarrow Y_{0}(p)\left(p^{r} \geqq 5\right)$.

$j(x) \quad \#\{$ ramification points

1728

0
2

2 if $p \equiv 1 \bmod 4$

if $p \equiv 1 \bmod 3$.

(1.2) The ramification points of $X_{0}(p) \longrightarrow X_{0}^{+}(p)(p \geqq 5)$ and $X_{0}\left(11^{2}\right) \longrightarrow$ $X_{0}^{+}\left(11^{2}\right)$.

Let $h=h(-p)$ be the class number of $\boldsymbol{Q}(\sqrt{-p})$, and $h^{\prime}=h^{\prime}(p)$ the class number of the order $Z[\sqrt{-p}]$ for $p \equiv 1 \bmod 4$. Then $h^{\prime}=h$ if $p \equiv-1$ $\bmod 8, h^{\prime} \equiv 3 h$ if $p \equiv 3 \bmod 8$ (see e.g., [12] Part 8 ). Denote by $s=s(p)$ 
the number of the ramification points of $X_{0}(p) \rightarrow X_{0}^{+}(p)(p \geqq 5)$. Then

$$
s= \begin{cases}h & \text { if } p \equiv 1 \bmod 4 \\ h+h^{\prime} & \text { if } p \equiv 1 \bmod 4\end{cases}
$$

(loc. cit.). Let $H$ (resp. $H^{\prime}$ ) be the Hilbert class field of $\boldsymbol{Q}(\sqrt{-p})$ (resp. of the order $Z[\sqrt{-p}]$ if $p \equiv 1 \bmod 4)$ and $x_{1}, \cdots, x_{h}, \cdots, x_{s}$ the ramification points. Let $H \stackrel{\iota}{\longrightarrow} C$ (resp. $H^{\prime} \stackrel{\iota^{\prime}}{\longrightarrow} C$ ) be an embedding, $\rho$ the complex conjugation of $H$ (resp. $H^{\prime}$ ) induced by this embedding, and $H^{+}$(resp. $H^{\prime+}$ ) the fixed field by $\rho$. For $i, 1 \leqq i \leqq h, x_{i}$ is defined over $H$ and cojugate over $Q(\sqrt{-p})$. One of them, say $x_{1}$, is defined over $H^{+}$. (Under the embedding c of $H$ into $C, x_{1}$ is represented by the elliptic curve $C / a$ for an ideal $\mathfrak{a}$ of the ring of integers of $\boldsymbol{Q}(\sqrt{-p})$ which satisfies $\left(\mathfrak{a}^{\rho}\right) \sim(\mathfrak{a})$ in the ideal class group of $Q(\sqrt{-p})$. If $p \equiv-1 \bmod 4, x_{h+i}\left(1 \leqq i \leqq h^{\prime}\right)$ are defined over $H^{\prime}$ and conjugate over $\boldsymbol{Q}(\sqrt{-p})$. One of them, say $x_{h+1}$, is defined over $H^{\prime+}$. (Under the embedding $\iota^{\prime}$ of $H^{\prime}$ into $C, x_{h+1}$ is represented by the elliptic curve $C / Z+Z \sqrt{-p}$ ).

There are six ramification points of $X_{0}\left(11^{2}\right) \rightarrow X_{0}^{+}\left(11^{2}\right)$, which are conjugate over $\boldsymbol{Q}$, and the set of the ramification points is a disjoint union of two orbits of $\operatorname{Gal}(\overline{\mathbf{Q}} / \boldsymbol{Q}(\sqrt{-\overline{1}}))$ of length three.

(1.3) The cuspidal sections of $X_{0}\left(p^{r}\right)$ ([2]) .

For integers $k, 1 \leqq k \leqq r$, and $i$ prime to $p$, let $\left(\begin{array}{c}i \\ p^{k}\end{array}\right)$ be the cuspidal section of $X_{0}\left(p^{r}\right)$ represented by the pair $\left(G_{m} \times Z / p^{r-k} Z, Z / p^{r} Z\left(\zeta^{i}, p^{k}\right)\right)$. Here, $\boldsymbol{Z} / p^{r} \boldsymbol{Z}\left(\zeta^{i}, p^{k}\right)$ is the cyclic subgroup of $\mu_{p^{r}} \times \boldsymbol{Z} / p^{r} \boldsymbol{Z}$ generated by $\left(\zeta^{i}, p^{k}\right), \zeta=\zeta_{p^{r}}$ is a primitive $p^{r}$-th root of 1 . We denote $0=\left(\begin{array}{l}0 \\ 1\end{array}\right)$ and $\infty=\left(\begin{array}{l}1 \\ 0\end{array}\right)$. The ramification index of the covering $X_{1}\left(p^{r}\right) \rightarrow X_{0}\left(p^{r}\right)$ at $\left(\begin{array}{c}i \\ p^{k}\end{array}\right)$ is $\min \left\{p^{k}, p^{r-k}\right\}$. Let $0_{i}, 1 \leqq i \leqq p^{r-1}(p-1)$, be the cuspidal sections of $X_{1}\left(p^{r}\right)$ lying over $0=\left(\begin{array}{l}0 \\ 1\end{array}\right)$, which are $\boldsymbol{Q}$-rational. We call them the 0 -cusps.

(1.4) We will use the following coverings. Here $\gamma$ is the generator of $\bar{\Gamma}_{0}\left(p^{r}\right) \simeq\left(Z / p^{r} Z\right)^{\times} / \pm 1, s=s(p)$ is the number of the ramification points of $X \rightarrow Y$, and $g(X)$ and $g(Y)$ are respectively the genuses of $X$ and $Y$. If $X=X_{0}(p)$ and $Y=X_{0}^{+}(p)$, put $g_{0}(p)=g(X), g_{+}(p)=$ $g(Y)$. 
Table 1.

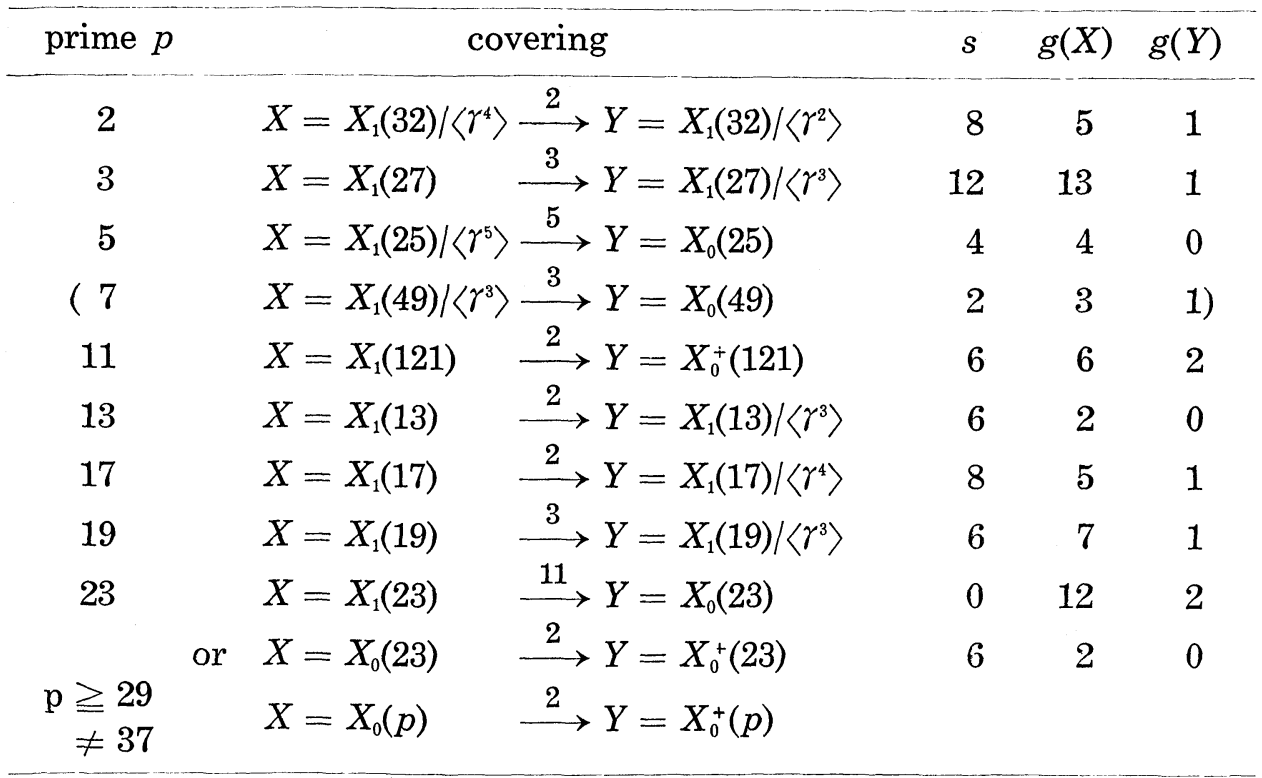

For $p=37$, let $\left(X_{1}(37) \stackrel{9}{\longrightarrow}\right) X \stackrel{2}{\longrightarrow} Y=X_{0}(37)$ be the double covering. Then $s=2, g(X)=4$ and $g(Y)=2$.

(1.5) Let $J=J(X)$ be the jacobian variety of the modular curve $X$ above. On the Mordell-Weil groups of $J$ or $J_{0}^{-}(p)(p \geqq 11)$, we know the following (Kenku [6, 8, 9], Mazur and Tate [20], Mazur [17], [35] Table 1, 3, 5).

Table 2.

\begin{tabular}{cl}
$p$ & \multicolumn{1}{c}{$\# J(\boldsymbol{Q})$ or $\# J_{0}^{+}(p)(\boldsymbol{Q})_{\text {tor }}$} \\
2 & $2 \cdot 5|\# J(\boldsymbol{Q})| 2^{9} \cdot 5^{2}$ \\
3 & $3 \cdot 19|\# J(\boldsymbol{Q})| 3^{4} \cdot 19 \cdot 307$ \\
5 & $J(\boldsymbol{Q}) \simeq Z / 71 Z$ \\
7 & $J(\boldsymbol{Q}) \simeq Z / 14 Z$ \\
11 & $2 \cdot 5\left|\# J_{0}^{-}(121)(\boldsymbol{Q})\right| 2^{a} \cdot 5^{2}$ for an integer $a \geqq 1$ \\
13 & $J(\boldsymbol{Q}) \simeq Z / 19 Z$ \\
17 & $2 \cdot 73|\# J(\boldsymbol{Q})| 2^{3} \cdot 73$ \\
19 & $3|\# J(\boldsymbol{Q})| 3^{2} \cdot 387$ \\
23 & $11\left|\# J_{1}(23)(\boldsymbol{Q})\right| 11 \cdot 37181$ \\
$p \geqq 11$ & $J_{0}^{-}(p)(\boldsymbol{Q})_{\text {tor }} \simeq Z / m Z$, where $m=$ num $((p-1) / 12)$. \\
\hline
\end{tabular}


For $p=37$, we will see that the Mordell-Weil group of Coker $\left(J_{0}(37) \rightarrow J(X)\right)$ is isomorphic to $Z / 5 Z$. (The double covering $X \rightarrow X_{0}(37)$ has two ramification points with the modular invariant $j=1728$ ).

(1.6) Let $\mathscr{X}_{1}\left(p^{r}\right), \mathscr{X}_{0}\left(p^{r}\right)$ be the normalizations of the projective $j$-line $\mathscr{X}_{0}(1) \simeq \boldsymbol{P}^{1}{ }_{Z}$ in $X_{1}\left(p^{r}\right)$ and $X_{0}\left(p^{r}\right)$, respectively. These are smooth over $Z[1 / p]$ ([2]VI Proposition (6.7)). The special fibre $\mathscr{X}_{1}\left(p^{r}\right) \otimes F_{p}$ (also $\left.\mathscr{X}_{0}\left(p^{r}\right) \otimes F_{p}\right)$ has $r+1$ irreducible components $E_{0}, \cdots, E_{r}$. The 0-cusps $\otimes \boldsymbol{F}_{p}$ are the sections of the smooth component $E_{0}^{h}=E_{0} \mid$ \{supersingular points on $\left.\mathscr{X}_{1}\left(p^{r}\right) \otimes \boldsymbol{F}_{p}\right\}$. Put $\mathscr{V}_{1}\left(p^{r}\right)=\mathscr{X}_{1}\left(p^{r}\right) \backslash \sum_{i=0}^{r-1} E_{i+1}$, which is smooth over $Z$. The 0 -cusps are the sections of $\mathscr{V}_{1}\left(p^{r}\right)$ ([2] $\mathrm{V} \S 2, \S 4, \mathrm{VI})$.

N.B. (loc. cit.). Let $\mathscr{C}^{\prime}=\mathscr{C}_{1}^{\prime}\left(p^{r}\right)$ be the algebraic stack which represents the functor: for a scheme $S / Z, \mathscr{C}^{\prime}(S)$ is the set of the isomorphism classes of the generalized elliptic curves $C$ with a $S$-section $P$ of order $p^{r}$ such that $\langle P\rangle \simeq\left(Z / p^{r} Z\right) / S$, isomorphic locally for the étale topology. Here $\langle P\rangle$ is the finite étale subgroup generated by the section $P$ (see loc. cit. $\mathrm{V} \S 2, \S 4)$. Let $\mathscr{V}_{1}\left(p^{r}\right)$ be the scheme induced by $\mathscr{C}^{\prime}$ (= "scherma grossier", loc. cit. VI, VII p. 300). Then $\mathscr{V}_{1}\left(p^{r}\right)$ is an open subscheme of $\mathscr{X}_{1}\left(p^{r}\right)$ and smooth over $Z$ (see loc. cit. $\mathrm{V} \S 2, \S 4, \mathrm{I}(8.22)$ ). The 0 -cusps are the sections of $\mathscr{V}_{1}\left(p^{r}\right)$ represented by the pairs $\left(G_{m} \times Z / p^{r} Z, \pm P\right)$ for $P \in Z / p^{r} Z$.

Let $k$ be an algebraic number field of degree $d, \tilde{k}$ the smallest Galois extension of $\boldsymbol{Q}$ containing $k$. For a rational prime $q$, let $q$ be a prime of $\tilde{k}$ lying over $q$. We denote by $f_{q}, e_{q}$ the degree of $q$ and the ramification index of $q$ in $\tilde{k}$, respectively. Let $C=C(k, p)$ be the set of rational primes $q$ as follows:

$$
\begin{gathered}
C(k, p)=\{q \neq 2, p\} \cup\left\{q=p \quad \text { if } e_{p}<p-1\right\} \\
\cup\{q=2 \quad \text { if } p \neq 2,11,17 \text { or } p \equiv 1 \bmod 8\} .
\end{gathered}
$$

Define an integer $n^{\prime}=n^{\prime}(k, p)$ as the least non-negative integer subjects to

$$
p^{n^{\prime}}>\min _{q \in C(k, p)}\left\{1+q^{f_{q}}+2 \sqrt{q^{f_{q}}}\right\}
$$

and

$$
n^{\prime}>4 \text { if } p=2, n^{\prime}>2 \text { if } p=3, n^{\prime}>1 \text { if } p=5,7
$$


For $p \geqq 23$, let $n^{\prime \prime}=n^{\prime \prime}(k, p)$ be the least integer such that $n^{\prime \prime} \geqq n^{\prime}$ and $p^{n^{\prime \prime}}>1+2^{f_{2}}+2 \sqrt{2^{f_{2}}}$. For the prime $p \equiv 1 \bmod 8(p \geqq 23), n^{\prime}=n^{\prime \prime}$ (see (1.7)).

For a $\tilde{k}$-rational point $x$ on $X_{1}\left(p^{r}\right)$ (resp. $\left.X_{0}\left(p^{r}\right)\right)$, by $x$ we denote the $\mathcal{O}_{\tilde{k}}$-section: Spec $\mathcal{O}_{\tilde{k}} \rightarrow \mathscr{X}_{1}\left(p^{r}\right)$ (resp. $\rightarrow \mathscr{X}_{0}\left(p^{r}\right)$ ) which is the unique extension of $x$. Let $E$ be an elliptic curve defined over $k$ with a $k$-rational point $P$ of order $p^{r}$, and $x$ the point on $Y_{1}\left(p^{r}\right)$ represented by the pair $(E, \pm P)$.

Lemma (1.9). Let $q$ be a rational prime such that $q \neq p$, or $q=p$ and $e_{q}<p-1$, and $\mathfrak{q}$ a prime of $\tilde{k}$ lying over $q$. If $p^{r}>1+q^{f q}+2 \sqrt{q^{f_{q}}}$, then $x^{\sigma} \otimes \mathcal{O} / \mathfrak{p}$ is a 0 -cusp for any $\sigma \in \operatorname{Isom}_{Q}(k, \bar{Q})$, where $\mathcal{O}$ is the ring of integers of $\tilde{k}$.

Proof. We denote $f_{q}$ by $f$, and $\mathscr{O}^{\text {ur }}$ the ring of integers of $\tilde{k} \otimes \boldsymbol{Q}_{q}^{\mathrm{ur}}$. The point $x^{\sigma}$ is represented by $\left(E^{o}, P^{o}\right)$ which is defined over $\tilde{k}$. By the universal property of the Néron model $E_{l o}$, there exists a homomorphism $f:\left(Z / p^{r} Z\right)_{10} \rightarrow E_{10}$ such that $f \otimes \tilde{k}$ is an isomorphism into $E$. Let $A$ be the flat closure of $f\left(\left(Z / p^{r} Z\right)_{10} \otimes \tilde{k}\right)$ in the Néron model $E_{/ 0}$, which is a finite flat group scheme of rank $p^{r}$. If $q \neq p, f$ is an isomorphism. If $q=p$ and $e_{p}<p-1$, by the fundamental property of the finite flat group schemes ([26] $\S 3$ Proposition (3.3.2)), $f$ is also an isomorphism $\left(: f \otimes \mathcal{O}^{\text {ur }}\right.$ is an isomorphism, then $\left.\operatorname{ker}\left(f \otimes \boldsymbol{F}_{q f}\right)=\{0\}\right)$. Since $p^{r}>1+q^{f}+2 \sqrt{q^{f}}$ ( $\geqq 5$ ), $E$ has semistable reduction at $\mathfrak{q}$ (Tate [35] p. 46), and has multiplicative reduction (e.g., [16] Lemma 2). Fix an embedding of $\tilde{k}$ into $\overline{\boldsymbol{Q}}_{q}$. Then the connected component $\left(E^{\sigma}{ }_{10} \otimes F_{q f}\right)^{0}$ of the unity is a torus $T$ and $T \otimes_{F_{q f} \boldsymbol{F}_{q^{2} f}} \simeq \boldsymbol{G}_{m / F_{q^{2}}}$. So if $x^{\sigma} \otimes \boldsymbol{F}_{q^{f}}$ is not a 0 -cusp, then $\boldsymbol{Z} / p^{r} \boldsymbol{Z} \subset$ $T\left(F_{q f}\right), \simeq Z /\left(q^{f}-1\right) Z$ or $\simeq Z /\left(q^{f}+1\right) Z$. Therefore the condition $p^{r}>$ $1+q^{f}+2 \sqrt{q^{f}}$ shows that $x^{\sigma} \otimes F_{q f}$ is a 0 -cusp.

(1.10) Now we describe the fixed points of $w=w_{p}$ of $\mathscr{X}_{0}(p) \otimes Z[1 / p]$ $(p \geqq 5)$.

Let $\mathscr{X}_{0}^{+}(p)$ be the quotient $\mathscr{X}_{0}(p) /\langle w\rangle$, which is smooth over $Z[1 / p]$. $\left(\mathscr{X}_{0}(p)\right.$ is smooth over $Z_{2}$ and the action of $w$ on $\mathscr{X}_{0}(p) \otimes F_{2}$ is generically étale of degree two, see [2] VI Proposition (6.7)). Let $q, \neq p$, be a rational prime, $y$ a fixed point of $w$ on $\mathscr{X}_{0}(p) \otimes F_{q}$. Then $y$ is represented by an elliptic curve $\left(\mid \bar{F}_{q}\right)$ with a subgroup $A$ of rank $p$ such that $(E, A) \simeq$ $\left(E / A, E_{p} / A\right)$ (see [2]). There exists an endomorphism $\alpha$ of $E$ such that 
$\alpha(A)=\{0\}$ and $\alpha^{2}=-p$. The pair $(E, \alpha)$ is lifted to characteristic zero (over a finite extension of $\boldsymbol{Q}_{q}^{\mathrm{ur}}$ ), see e.g., [12] Part $12 \S 5$ Theorem 14). Thus $y$ is the special fibre of a fixed point $x_{i}$ for an integer $i, 1 \leqq i$ $\leqq s=s(p)$ (see (1.2)). Let $x$ be a fixed point of $w$ on $X_{0}(p)$ and $\mathcal{O}$ the ring of integers of $\boldsymbol{Q}_{q}^{\mathrm{ur}}$. Let $\widehat{\mathcal{O}_{\mathscr{x}_{0}(p), x}}=\mathcal{O}[[t]]$ be the completion along the O-section $x$ ([2] VI Proposition (6.7)). Then, $\sigma=w^{*}$ is of the form $\sigma(t)=-t+a_{2} t^{2}+\cdots$ for $a_{i} \in \mathcal{O}$ and $a_{j} \in \mathcal{O}^{\times}$for some $j$ if $q=2$. If $q \neq 2$, $p$, it is easily seen that $x_{i} \otimes \overline{\boldsymbol{F}}_{q} \neq x_{j} \otimes \overline{\boldsymbol{F}}_{q}$ for $x_{i} \neq x_{j}$.

Now assume $q=2(p \geqq 5)$. The double covering $\mathscr{X}_{0}(p) \otimes F_{2} \rightarrow$ $\mathscr{X}_{0}^{+}(p) \otimes \boldsymbol{F}_{2}$ has wild ramifications at the fixed points of $w=w \otimes \boldsymbol{F}_{2}$ (see e.g., [29] Chapitre IV). By the Riemann-Hurwitz formula, $2 g_{0}(p)-2=$ $2\left(2 g_{+}(p)-2\right)+\sum_{y}(1+i(y))$, where $y$ are the ramification points and $i(y)$ is the index of wild ramification at $y$ (see loc. cit., [17] Chapter II). Therefore, there are at most $s(p) / 2$ ramification points on $\mathscr{X}_{0}(p) \otimes \overline{\boldsymbol{F}}_{2}$. Let $v=v_{2}$ be the normalized valuation of $\overline{\boldsymbol{Q}}_{2}$ such that $v(2)=1$.

Sublemma. Let $x, \sigma$ and $\mathcal{O}$ be as above, and $\pi$ a prime element of $\mathcal{O}$. Let $\mathcal{O}^{\prime}$ be the ring of integers of the cyclic extension of $\boldsymbol{Q}_{2}^{\mathrm{ur}}(x)$ of degree three, and $\pi^{\prime}$ a prime element of $\mathcal{O}^{\prime}$.

(i) If $v(\pi)=1\left(\mathcal{O} \simeq W\left(\bar{F}_{2}\right)\right)$, there are at most two solutions $t=\alpha \in \pi \mathcal{O}$ of $t=\sigma(t)$, and at most three solutions $t=\alpha \in \pi^{\prime} \mathcal{O}^{\prime}$ of the same equation.

(ii) If $v(\pi)=1 / 2, t=\sigma(t)$ has at most two solutions in $\pi \mathcal{O}$.

Proof. The relation $\sigma^{2}=1$ implies $a_{3}=-a_{2}^{2}$. The remaining part is elementary.

Case $p \equiv 1 \bmod 8$. The ramification index of the rational prime 2 in $H$ is 2 (see (1.2)). By (ii) above we see that the map $\left\{x_{i}\right\} \rightarrow\left\{x_{i} \otimes \bar{F}_{2}\right\}$ is two to one. Two of $x_{i} \otimes \overline{\boldsymbol{F}}_{2}$ are $\boldsymbol{F}_{2}$-rational (see [24] Theorem 3).

Case $p \equiv 5 \bmod 8$. By the same reason as above, the map $\left\{x_{i}\right\} \rightarrow$ $\left\{x_{i} \otimes \overline{\boldsymbol{F}}_{2}\right\}$ is two to one. One of $x_{i} \otimes \bar{F}_{2}$ is $\boldsymbol{F}_{2}$-rational (loc. cit.).

Case $p \equiv-1 \bmod 8$. In this case $H=H^{\prime}$ (see (1.2)), the rational prime 2 splits in $\boldsymbol{Q}(\sqrt{-p})$ and $x_{i} \otimes \overline{\boldsymbol{F}}_{2}$ are not the supersingular points (e.g., [13] Chapter 8, [30]). By the uniqueness of the Deuring lifting (e.g., [12] Part 13, $\S 4$ Theorem 13), $\left\{\boldsymbol{x}_{i}\right\}_{1 \leqq i \leqq h} \rightarrow\left\{\boldsymbol{x}_{i} \otimes \overline{\boldsymbol{F}}_{2}\right\}$ is injective. Hence (i) above shows that the map $\left\{x_{i}\right\}_{1 \leqq i \leqq 2 h} \rightarrow\left\{x_{i} \otimes \bar{F}_{2}\right\}$ is two to one. Let $\mathfrak{p}=\mathfrak{p}_{2}$ be a prime of $H$ lying over 2. Then these $\left\{x_{i} \otimes \bar{F}_{2}\right\}$ is the disjoint union of orbits of the action of $\operatorname{Gal}\left(H_{p} / Q(\sqrt{-p})\right) \simeq \operatorname{Gal}\left(\kappa(p) / F_{2}\right)$. Here $H_{p}$ is the 
$\mathfrak{p}$-adic completion of $H$ and $\kappa(\mathfrak{p})=\mathcal{O}_{H} / \mathfrak{p}$. Note that the degree of $\mathfrak{p}$ is odd $\geqq 3$ for $p \geqq 23, p \equiv-1 \bmod 8$.

Case $p \equiv 3 \bmod 8$. The rational prime 2 does not ramify in $H$ and the degree of the prime $\mathfrak{p} \mid 2$ of $H$ is two. $H^{\prime}$ is a cyclic extension of $H$ of degree 3, which ramifies totally at the primes lying over 2 (e.g., [12] Part 8 Theorem 7). Then $x_{i}{ }^{\sigma} \otimes \kappa\left(\mathfrak{p}^{\prime}\right)=x_{i} \otimes \kappa\left(\mathfrak{p}^{\prime}\right)$ for a prime $\mathfrak{p}^{\prime} \mid 2$ of $H^{\prime}$ and $\sigma \in \operatorname{Gal}\left(H^{\prime} \mid H\right)$, where $\kappa\left(\mathfrak{p}^{\prime}\right)=\mathcal{O}_{H^{\prime}} / \mathfrak{p}^{\prime}$. Let $E / \boldsymbol{F}_{2}$ be a supersingular elliptic curve. Then $x_{i} \otimes \overline{\boldsymbol{F}}_{2}$ is represented by the pair $(E, A)$ for $A=$ $\operatorname{ker}(\alpha: E \rightarrow E), \alpha^{2}=-p$. Under the isomorphism

$$
\text { End }(E) \stackrel{\sim}{\longrightarrow}\left\{\frac{a+b i+c j+d k}{2} \mid a, b, c, d \in Z, a \equiv b \equiv c \equiv d \bmod 2\right\}
$$

(e.g., [35] §7), $\alpha$ is represented by $a i+b j+c k$ for $a, b, c \in Z$. Then, as $p \equiv 3 \bmod 8, a, b, c$ must be odd. Therefore $A$ is invariant under the action of $(1+\alpha) / 2 \in$ End $(E)$. Let $(\tilde{E}, \tilde{\beta})$ be a lifting of $(E,(1+\alpha) / 2)$ (e.g., [12] Part 13, $\S 5$ Theorem 14). Then $x_{i} \otimes \bar{F}_{2}$ is the special fibre of $x_{j}$ for a $j, 1 \leqq j \leqq h$, see (1.2). $\quad x_{j}$ is represented by $(\tilde{E}, \operatorname{ker}(2 \tilde{\beta}-1))$. Thus we see that the map $\left\{x_{i}\right\}_{1 \leqq i \leqq h} \rightarrow\left\{x_{i} \otimes \bar{F}_{2}\right\}$ is one to one (see (i) above), and $\left\{x_{i+h}\right\}_{1 \leqq i \leqq 3 h} \rightarrow\left\{x_{i} \otimes \bar{F}_{2}\right\}$ is three to one. One of $x_{i} \otimes \overline{\boldsymbol{F}}_{2}$ is $\boldsymbol{F}_{2}$-rational ([24] Theorem 3).

Let $y_{j}$ be the fixed point of $w=w \otimes F_{2}$ on $\mathscr{X}_{0}(p) \otimes \boldsymbol{F}_{2}(p \geqq 5), \quad i(y)$ be the index of the wild ramification at $y_{j}$ of the natural morphism $\mathscr{X}_{0}(p) \otimes \boldsymbol{F}_{2} \rightarrow \mathscr{X}_{0}^{+}(p) \otimes \boldsymbol{F}_{2}$.

Table 3.

\begin{tabular}{rccc}
\hline$p \bmod 8$ & $i\left(y_{j}\right)$ & $\#\left\{\boldsymbol{F}_{2}\right.$-rational fixed points $\}$ & $\#\left\{\right.$ non $\boldsymbol{F}_{2}$-rational fixed points $\}$ \\
\hline 1 & 1 & 2 & $h / 2-2$ \\
5 & 1 & 1 & $h / 2-1$ \\
-1 & 1 & $0(p \geqq 23)$ & $h(p \geqq 23)$ \\
3 & 3 & 1 & $h-1$
\end{tabular}

Let $K$ be a field, $X$ a proper smooth curve defined over $K$. Let $\sigma \neq 1$ be an automorphism of $X$ defined over $K,\left\{x_{i}\right\}_{1 \leqq i \leqq s}$ the set of the fixed points of $\sigma$, and set $D=\sum_{i=1}^{s}\left(x_{i}\right)$ a divisor of $X$. It is easy to see the following.

LEMMa (1.11). If $g$ is a rational function on $X$ of degree $m$ defined over $K$ such that $\left(\sigma^{*} g\right) \neq(g)(=$ the divisor of $g)$ and $g\left(x_{i}\right) \neq 0, \infty$. Then 


$$
\left(\sigma^{*} g / g-1\right)_{0}>D
$$

In particular, $s \leqq 2 m$. If, moreover, $\sigma^{2}=1$,

$$
\left(\sigma^{*} g / g-1\right)_{0}=\sum_{i=1}^{s} m_{i}\left(x_{i}\right)+\sum_{j}\left\{\left(y_{j}\right)+\left(\sigma y_{j}\right)\right\}
$$

for some positive integers $m_{i}$ such that $\sum_{i=1}^{s} m_{i}\left(x_{i}\right)$ is K-rational and $y_{j} \neq \sigma y_{j}$.

Now let $K$ be a finite extension of $\boldsymbol{Q}_{2}, R$ the ring of integers of $K$ with the residue field $\boldsymbol{F}_{q}$ for $q=2^{r}$. Suppose that $X$ is the generic fibre of a smooth projective curve $\mathscr{X} \rightarrow$ Spec $R$, and $\sigma$ an involution of $\mathscr{X}$ defined over $R$ such that $\mathscr{Y}_{\mathrm{dfn}}=\mathscr{X} \mid\langle\sigma\rangle \rightarrow \operatorname{Spec} R$ is smooth and that the natural morphism $f: \mathscr{X} \otimes \boldsymbol{F}_{q} \rightarrow \mathscr{Y} \otimes \boldsymbol{F}_{q}$ is not radicial. Let $E=\sum m_{i}\left(z_{i}\right)$, $m_{i}>0$, be a $K$-rational divisor of $X$ such that $1<\operatorname{dim}_{K} H^{0}(X, \mathcal{O}(E))$. Then we have

Lemma (1.12). Assume further that $\sigma=\sigma \otimes \boldsymbol{F}_{q}$ has fixed points, $\boldsymbol{z}_{i} \otimes \boldsymbol{F}_{q}$ are not fixed points and that $\sigma^{*}\left(\sum m_{i}\left(z_{i} \otimes \boldsymbol{F}_{q}\right)\right)=\sum m_{i}\left(z_{i} \otimes \boldsymbol{F}_{q}\right)$. Then there exists a covering $g: \mathscr{Y} \otimes \boldsymbol{F}_{q} \rightarrow \boldsymbol{P}^{1}{ }_{\boldsymbol{F}_{q}}$ defined over $\boldsymbol{F}_{q}$ such $f^{*}\left((g)_{\infty}\right)>$ $\sum m_{i}\left(\boldsymbol{z}_{i} \otimes \boldsymbol{F}_{q}\right)$.

Proof. Let $K^{\prime}$ be a finite extension of $K$ over which the $z_{i}$ 's are defined, and $R^{\prime}, F_{q^{\prime}}$ the ring of integers of $K^{\prime}$ and the residue field of $R^{\prime}$, respectively. Let $\mathscr{L}=\otimes^{i} \mathcal{O}\left(z_{i}\right)^{\otimes m_{i}}$ be the Cartier divisor of $\mathscr{X} \otimes R^{\prime}$. Then $\operatorname{dim}_{F_{q^{\prime}}} H^{0}\left(\mathscr{X} \otimes F_{q^{\prime}}, \mathscr{L}\right)>1$ by the upper semicontinuity ([34] (7.7.5)1). Then $\operatorname{dim}_{F q} H^{0}\left(\mathscr{X} \otimes F_{q}, \mathcal{O}(E)\right)>1$, because $\mathscr{L} \simeq \mathcal{O}(E) \otimes R^{\prime}$ over $\mathscr{X} \otimes R^{\prime}$. By the assumption $\sigma^{*}\left(E \otimes F_{q}\right)=E \otimes F_{q}$, there exists a non-constant section $h$ of $H^{0}\left(\mathscr{X} \otimes \boldsymbol{F}_{q}, \mathcal{O}(E)\right)$ such that $\boldsymbol{F}_{q} \oplus \boldsymbol{F}_{q} h$ is a $\sigma$-invariant subspace. So $\sigma^{*} h=$ $h+a$ for an $a \in \boldsymbol{F}_{q}$. The proof is completed if $a=0$ is shown (because $\mathscr{X} \otimes \boldsymbol{F}_{q} \rightarrow \mathscr{Y} \otimes \boldsymbol{F}_{q}$ is generically étale of degree 2). Suppose $a \neq 0$. For each point $x$ on $\mathscr{X} \otimes \boldsymbol{F}_{q} \backslash \operatorname{Supp}\left(E \otimes \boldsymbol{F}_{q}\right), h \in \mathcal{O}_{\mathscr{x} \otimes \boldsymbol{F}_{q}, x}$. The covering $\mathscr{X} \otimes \boldsymbol{F}_{q} \rightarrow$ $\mathscr{Y} \otimes \boldsymbol{F}_{q}$ is then factored by $\operatorname{Spec} \mathcal{O}_{\mathscr{y} \otimes \boldsymbol{F}_{q}, f(x)}[h]$ at $x$ :

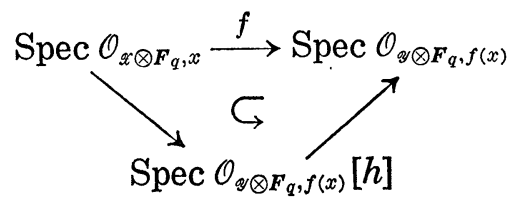

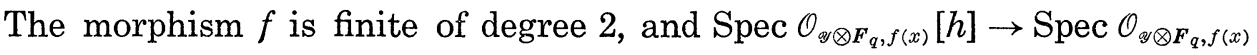
is étale of degree 2, since $\sigma^{*} h(z) \neq h(z)$ for any point $z$ on $\mathscr{X} \otimes \boldsymbol{F}_{q} \mid$ $\operatorname{Supp}\left(E \otimes \boldsymbol{F}_{q}\right)$. Therefore $f$ is étale at any point $x \in \mathscr{X} \otimes \boldsymbol{F}_{q} \mid \operatorname{Supp}\left(E \otimes \boldsymbol{F}_{q}\right)$. 
This contradicts to our assumption.

\section{§. The rational points on $Y_{1}\left(p^{r}\right)$}

Let $k$ be an algebraic number field of degree $d ; \tilde{k}, e_{q}, f_{q}, C=C(k, p)$, $n^{\prime}=n^{\prime}(k, p)$ and $n^{\prime \prime}=n^{\prime \prime}(k, p)$ be as in the last section. Assuming the existence of a $k$-rational point on $Y_{1}\left(p^{r}\right)$ with $r>n(k, p)$, we here introduce a rational function $g$ on a modular curve whose divisor is determined by the $k$-rational point as above. Further we prepare propositions which concern $g$ and the fixed points of $w_{p}(p \geqq 23)$. Let $x$ be a $k$-rational point on $Y_{1}\left(p^{r}\right)=X_{1}\left(p^{r}\right) \backslash\{$ cusps $\}$ for $r \geqq n^{\prime}(k, p)$. By $x$ we denote also the image of $x$ by the natural morphism $X_{1}\left(p^{r}\right) \rightarrow X$, see (1.4). We consider only the primes $p$ with $p \leqq 23$ or $\left(p \geqq 29\right.$ and) $\# J_{0}^{-}(p)(\boldsymbol{Q})<\infty$. For each $\sigma \in \operatorname{Isom}_{Q}(k, \bar{Q})$, Lemma (1.9) shows that $x^{\sigma} \otimes \kappa(\mathfrak{q})=0_{i_{\sigma}} \otimes \kappa(\mathfrak{q})$ for an integer $i_{\sigma}$ and a prime $q$ of $\tilde{k}$ lying over the rational prime $q \in C=C(k, p)$ which attains the minimal value of $1+q^{f q}+2 \sqrt{q^{f q}}$, where $\kappa(\mathfrak{q})=\mathcal{O}_{\tilde{k}} / \mathfrak{q}$. Consider the $\boldsymbol{Q}$-rational section

$$
i(x)=\operatorname{cl}\left(\sum_{\sigma}\left(x^{\sigma}\right)-\sum_{\sigma}\left(0_{i_{\sigma}}\right)\right)
$$

of $A=J(X)$ for $p \leqq 23, p \neq 11$; of $A=\operatorname{Coker}\left(J_{0}(37) \rightarrow J(X)\right)$ for $p=37$; of $A=J_{0}^{-}(121)$ for $p=11$; and of $A=J_{0}^{-}(p)$ for $p \geqq 29$ (see (1.4)). Let $\mathscr{X}$ be the normalization of the projective $j$-line $\mathscr{X}_{0}(1)$ in $X$ (see (1.4)). Let $\boldsymbol{Z}_{(q)}$ be the localization of $\boldsymbol{Z}$ at the prime $q$ and $\mathcal{O}_{(q)}=\mathcal{O}_{\tilde{k}} \otimes \boldsymbol{Z}_{(q)}$. Then $x^{\sigma} \otimes \mathcal{O}_{(q)}, 0_{i_{\sigma}} \otimes \mathcal{O}_{(q)}$ are the sections of the smooth part of $\mathscr{X} \otimes Z_{(q)}$, see (1.6), (1.9). Let

$$
i\left(x^{\sigma}\right): \operatorname{Spec} \mathcal{O}_{(q)} \stackrel{x^{\sigma}}{\longrightarrow} \mathscr{X}^{\text {smooth }} \otimes Z_{(q)} \longrightarrow A_{/ Z_{(q)}} \quad z \longrightarrow \operatorname{cl}\left((z)-\left(0_{i_{\sigma}}\right)\right) .
$$

Then by our assumptions on $q$ and $r$ (see (1.8), (1.9)), $i\left(x_{\sigma}\right) \otimes \kappa(q)=0$. Then $i(x) \otimes \kappa(\mathfrak{q})=\left(\sum_{\sigma} i\left(x^{j}\right)\right) \otimes \kappa(\mathfrak{q})=0$, i.e., $i(x) \otimes \boldsymbol{F}_{q}=0$. The $\boldsymbol{Q}$-rational section $i(x) \otimes Z_{(q)}$ is of finite order for $p \neq 37$, see (1.5). The specialization lemma of the finite flat group schemes ([26] Proposition (3.3.2), [18] Proposition (1.2)) leads that $i(x)=0$ for $p \neq 37$ (, note: $1<3-1 \leqq q-1$, (1.7)). Then there is a rational function $g$ on $X$ such that (see (1.4))

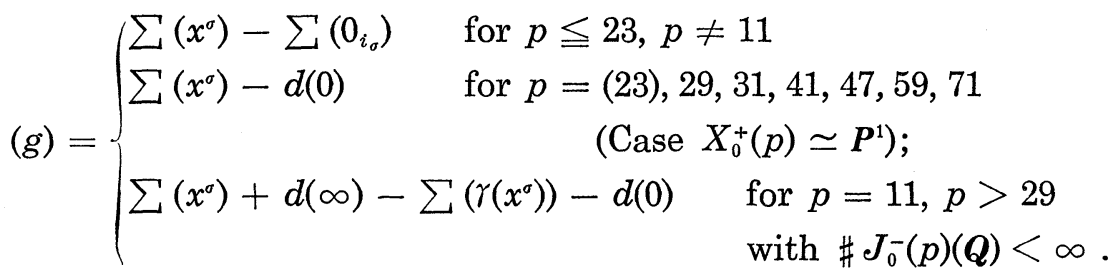


For $p=37$, we will show $\operatorname{Coker}\left(J_{0}(37) \rightarrow J(X)\right)(\boldsymbol{Q}) \simeq Z / 5 Z$, see (3.4.2). Then we get a rational function $g$ on $X$ such that

$$
(g)=\sum\left(x^{\sigma}\right)+\sum\left(\gamma\left(0_{i_{\sigma}}\right)\right)-\sum\left(\gamma\left(x^{\sigma}\right)\right)-\sum\left(0_{i_{\sigma}}\right),
$$

where $1 \neq \gamma \in \operatorname{Aut}\left(X / X_{0}(37)\right)$, see (1.4). As (g) is $\boldsymbol{Q}$-rational, we may assume that $g$ is defined over $\boldsymbol{Q}$. If $p=11$ or $p$ is the last case in (2.1), $\left(w^{*} g\right)=-(g)(\neq 0)$; and if $p=37,\left(\gamma^{*} g\right)=-(g)$. So we may assume

$$
\left\{\begin{array}{l}
w^{*} g=\frac{a}{g} \\
\gamma^{*} g=\frac{a}{g} \quad(\text { for } p=37)
\end{array}\right.
$$

for a square free integer $a(\neq 0)$. For $p \neq 37$, as $Q\left(x_{i}\right)$ is not totally imaginary (see (1.2)), $a>0$.

Proposition (2.3). Let $x$ be a k-rational point on $Y_{1}\left(p^{r}\right)$, g the rational function as above and $p=2,3,11,17$, or $p \geqq 23, \neq 37$, with $\# J_{0}^{-}(p)(\boldsymbol{Q})<\infty$. In the case $p \equiv 5 \bmod 8$ and the class number $h=h(-p)$ of $\boldsymbol{Q}(\sqrt{-p})$ is divisible by 4 , we further assume $p^{r}>1+q^{f_{q}}+2 \sqrt{q^{f_{q}}}$ for an odd prime $q \neq p$. Then we have

$$
s=s(p) \leqq 2 d .
$$

Proof. Case $p \geqq 23$ and $X_{0}^{+}(p) \simeq P^{1}$.

The rational function $g$ is of degree $d$ and $(g) \neq\left(w^{*} g\right)$. So the conditions of Lemma (1.11) are satisfied.

Case $p \geqq 23, \neq 37$, and $X_{0}^{+}(p) \neq P^{1}$.

Let $x_{1}, \cdots, x_{h}, \cdots, x_{s}$ be the fixed points of $w=w_{p}$. Then $g\left(x_{i}\right)= \pm \sqrt{a}$ (see (2.2)). We may assume $g\left(x_{1}\right)=+\sqrt{a}$. First, we consider the case $p \equiv-1 \bmod 4$. Then $s=s(p)=h+h^{\prime}$ (see (1.4)) is even, and $h\left(\leqq h^{\prime}\right)$ is odd. $x_{1}$ is defined over $H^{+}$(see (1.4)) and $\left[H^{+}: Q\right]$ is odd, so that $a=1$ (by our choice of $a$, see (2.2)). The points $x_{1}, \cdots, x_{h}\left(\operatorname{resp} . x_{h+1}, \cdots, x_{h+h^{\prime}}\right.$ ) are conjugate to each other over $\boldsymbol{Q}$, so that

$$
(g-1)_{0}>\sum_{i=1}^{h}\left(x_{i}\right)
$$

and

$$
(g-1)_{0}>\sum_{i=1}^{h^{\prime}}\left(x_{h+i}\right) \quad \text { or }(g+1)_{0}>\sum_{i=1}^{h^{\prime}}\left(x_{h+i}\right) .
$$

In the first case $s=h+h^{\prime} \leqq 2 d$. In the second case, Lemma (1.11) and 
and the fact that $h$ and $h^{\prime}$ are odd integers show

$$
(g-1)_{0}>2 \sum_{i=1}^{h}\left(x_{i}\right)
$$

and

$$
(g+1)_{0}>2 \sum_{i=1}^{h^{\prime}}\left(x_{h+i}\right) .
$$

Thus $2 d \geqq 2 h^{\prime} \geqq s$.

Next, we consider the case $p \equiv 1 \bmod 4$. If $2 d<h=s$, then

$$
\begin{aligned}
& (g-\sqrt{a})_{0}>\Sigma^{\prime}\left(x_{i}\right) \\
& (g+\sqrt{a})_{0}>\Sigma^{\prime \prime}\left(x_{i}\right)
\end{aligned}
$$

where $\sum^{\prime}+\sum^{\prime \prime}=\sum_{i=1}^{s}$ and $a>1$ (because $x_{i}$ are conjugate to each other over $\boldsymbol{Q})$. If $h \neq \equiv \bmod 4$, our assumption and Lemma (1.11) show $(g-\sqrt{a})_{0}>$ $2 \sum^{\prime}\left(x_{i}\right)$ and $(g+\sqrt{a})_{0}>2 \sum^{\prime \prime}\left(x_{i}\right)$. This contradicts that $s>2 d$. If $h \equiv 0$ $\bmod 4, a=p$. Set $D^{\prime}=\sum^{\prime}\left(x_{i}\right) . \quad D^{\prime}$ is a divisor of degree $s / 2$ and

$$
(g-\sqrt{p})=D^{\prime}+E-\sum_{\sigma}\left(w\left(x^{\sigma}\right)\right)-d(0),
$$

for an effective divisor $E$. We have $w^{*} E=E$. By the assumption, there is an odd prime $q \neq p$ such that $p^{r}>1+q^{f_{q}}+2 \sqrt{q^{f_{q}}}$. Using the upper semicontinuity ([34] (7.7.5), 1), we get a rational function $f$ on $\mathscr{X}_{0}(p) \otimes \overline{\boldsymbol{F}}_{q}$ such that

$$
(f)=D^{\prime}+E-d(\infty)-d(0) .
$$

Then $\left(w^{*} f\right)=(f)$ so $w^{*} f= \pm f$. If $w^{*} f=+f, E>D^{\prime}$. If $w^{*} f=-f,(f)_{0}>$ $D=\sum_{i=1}^{s}\left(x_{i}\right)$ (see (1.11)). Thus $s \leqq 2 d$.

Case $p=11$. The number of the fixed points of $w=w_{121}$ on $X_{0}(121)$ is six. Using $g$ in (2.1), (2.2), we get $d \geqq 3=s / 2$ by the same way as above.

Case $p=2$, 17. Let $f=\gamma^{*} g / g$ for $1 \neq \gamma \in \operatorname{Gal}(X / Y)$ (see (1.4)). Then $\gamma^{*} f=1 / f$ and $(f)=\sum\left(\gamma\left(x^{\sigma}\right)\right)+\sum\left(0_{i_{\sigma}}\right)-\sum\left(x^{\sigma}\right)-\sum\left(\gamma\left(0_{i_{\sigma}}\right)\right)$. If $\left(\gamma^{*} g\right)=(g)$, then $\gamma\left(x^{\sigma}\right)=x^{\epsilon}$ for an $\iota \in \operatorname{Isom}_{Q}(k, \overline{\mathbf{Q}})$ and any $\sigma \in \operatorname{Isom}_{Q}(k, \overline{\mathbf{Q}})$. If $d=2$, we see that $\left\{x, x^{\sigma}=\gamma(x)\right\}$ defines a $Q$-rational point on $Y$. But we know that the $\boldsymbol{Q}$-rational points on $X_{0}(32)$, and on $X_{0}(17)$ are the cuspidal points ([35] table 1). If $d=3$, one of the $x^{\sigma}$ becomes a fixed point of $\gamma$. But we know that a ramification point of $X \rightarrow Y$ is either a cuspidal point or a point with the modular invariant $j=1728$, see (1.1), (1.4). Therefore 
$\left(\gamma^{*} g\right) \neq(g)$ for $d=2$ and 3. The rest then follows from Lemma (1.11).

Case $p=3$. Let $f=\gamma^{*} g / g$ for $1 \neq \gamma \in \operatorname{Gal}(X / Y)$ (see (1.4)). Then $\left.(f)=\sum\left(\gamma\left(x^{\sigma}\right)\right)+\sum\left(0_{i_{\sigma}}\right)\right)-\sum\left(x^{\sigma}\right)-\sum\left(\gamma\left(0_{i_{\sigma}}\right)\right)$. For $d<6=s / 2$, if $\left(\gamma^{*} g\right)=$ (g), $\gamma\left(x^{\sigma}\right)=x^{c \sigma}$ for an $\iota \in \operatorname{Isom}_{Q}(k, \overline{\mathbf{Q}})$ and any $\sigma \in \operatorname{Isom}_{Q}(k, \overline{\mathbf{Q}})$. Any fixed point of $\gamma$ is a cuspidal point or a point with the modular invariant $j=0$, so that $\gamma\left(x^{\sigma}\right) \neq x^{\sigma}$ for any $\sigma \in \operatorname{Isom}_{Q}(k, \bar{Q})$, see (1.4). Then $\left\{x^{\sigma}\right\}_{\sigma}$ is a disjoint union of $\langle\gamma\rangle$-orbits of length 3. If $d=3,\left\{x^{\sigma}\right\}_{\sigma}=\left\{x, \gamma(x), \gamma^{2}(x)\right\}$ defines a $\boldsymbol{Q}$-rational point on $Y$. But a $\boldsymbol{Q}$-rational point on $X_{0}(27)$ is a cuspidal point or a point with the modular invariant $j=0$ (see (1.4), [35] table 1). Therefore $\left(\gamma^{*} g\right) \neq(g)$ for $d<6$. Then by Lemma (1.11) we get the result.

Let $\mathscr{X}^{+}=\mathscr{X}_{0}^{+}(p)$ be the quotient $\mathscr{X}_{0}(p) /\left\langle w_{p}\right\rangle$, which is smooth over $Z[1 / p]$ (see $(1.10))$.

Proposition (2.4). Let $p \geqq 23, \neq 37$ be a prime number satisfying the condition $\# J_{0}^{-}(p)(Q)<\infty$, g the rational function on $X_{0}(p)$ in (2.2). If $p^{r}>1+2^{f_{2}}+2 \sqrt{2^{f_{2}}}$, then there is a covering $f$ defined over $\boldsymbol{F}_{2}$,

$$
\mathscr{X}^{+} \otimes \boldsymbol{F}_{2} \stackrel{f}{\longrightarrow} \boldsymbol{P}^{1}{ }_{/ \boldsymbol{F}_{2}}
$$

such that $(f)_{\infty}=d^{\prime}$ (cusp) for an integer $d^{\prime}, 1 \leqq d^{\prime} \leqq d$.

Proof. Let $\mathscr{L}=\left(\otimes^{\sigma} \mathcal{O}\left(x^{\sigma}\right)\right) \otimes \mathcal{O}(d(\infty))$ be the Cartier divisor on $\mathscr{X}_{0}(p) \otimes \mathcal{O}_{\tilde{k}}$, where $\mathscr{O}_{\tilde{k}}$ is the ring of integers of $\tilde{k}$. By our assumption, $\operatorname{dim}_{\bar{F}_{2}} H^{0}\left(\mathscr{X}_{0}(p) \otimes \bar{F}_{2}, \mathscr{L}\right)$ $>1$ (see [34] (7.7.5), 1), and $\mathscr{L} \otimes \overline{\boldsymbol{F}}_{2}=\mathcal{O}(d(0)+d(\infty))$, see (1.9). The cusps $0=0 \otimes F_{2}$ and $\infty=\infty \otimes F_{2}$ are not the fixed points of $w=w \otimes \boldsymbol{F}_{2}$, while $\mathscr{X}_{0} \otimes \boldsymbol{F}_{2} \rightarrow \mathscr{X}^{+} \otimes \boldsymbol{F}_{2}$ has ramifications points. The divisor $d(0)+d(\infty)$ is $F_{2}$-rational, and is $w$-invariant. So Lemma (1.12) yields the desired covering $f$.

Corollary (2.5). Under the assumption of (2.4),

$$
\begin{gathered}
\sharp \mathscr{X}_{0}^{+}(p)\left(F_{2^{m}}\right)<1+2^{m} d \\
\# \mathscr{X}_{0}(p)\left(F_{4}\right) \leqq 2+8 d-s(p) .
\end{gathered}
$$

Proposition (2.6). Let $p \geqq 23, \neq 37$, be a prime number such that $\#_{0} J_{0}^{-}(p)(\boldsymbol{Q})<\infty$. Assume that $r \geqq n^{\prime \prime}=n^{\prime \prime}(k, p)$ (see (1.8)) and let $g$ be the rational function on $X_{0}(p)$ in (2.2). Then we get the following estimates of $\#^{*} \mathscr{X}_{0}^{+}(p)\left(\boldsymbol{F}_{2 m}\right)$.

(i ) $p \equiv 1 \bmod 8 ; \# \mathscr{X}_{0}^{+}(p)\left(\boldsymbol{F}_{2}\right) \leqq 2+2 d-h / 4$. 
(ii) $p \equiv 5 \bmod 8$ and $h=h(-p) \not \equiv 0 \bmod 4$;

$$
\text { or } \quad \begin{aligned}
& \quad \mathscr{X}_{0}^{+}(p)\left(F_{2}\right) \leqq 2+2 d-h / 2 \\
& \quad \# \mathscr{X}_{0}^{+}(p)\left(F_{4}\right) \leqq 1+4 d-(h-2) / 4 .
\end{aligned}
$$

(iii) $p \equiv-1 \bmod 8 ; \# \mathscr{X}_{0}^{+}(p)\left(F_{2}\right) \leqq 1+2 d-h$

and

$$
\# \mathscr{X}_{0}^{+}(p)\left(F_{4}\right) \leqq 1+4 d-h .
$$

(iv) $p \equiv 3 \bmod 8 ; \# \mathscr{X}_{0}^{+}(p)\left(F_{2}\right) \leqq 2+2 d-2 h$

and

$$
\# \mathscr{X}_{0}^{+}(p)\left(\boldsymbol{F}_{4}\right) \leqq 1+4 d-h .
$$

Proof. Let $x_{1}, \cdots, x_{s}$ (resp. $y_{1}=x_{1} \otimes F_{2}, y_{2}, \cdots, y_{h / 2}$ if $p \equiv 1 \bmod 4$; $y_{1}, y_{2}, \cdots, y_{h}$ if $\left.p \equiv-1 \bmod 4\right)$ be the fixed points of $w=w_{p}$ on $X_{0}(p)$ (resp. $\mathscr{X}_{0}(p) \otimes F_{2}$ ), see (1.10). Then $g\left(x_{i}\right)= \pm \sqrt{a}$ (see (2.3)). We may assume $g\left(x_{1}\right)=+\sqrt{a} \in H^{+}$(see (1.2)). As in the proof of (2.3), $a=1$, or $a=p$ if $p \equiv 1 \bmod 4$. Set $D=\sum_{i=1}^{s}\left(x_{i}\right)$.

Case $p \equiv 1 \bmod 4$ and $a=1$. The divisor of $g-1$ is

$$
(g-1)=D+E-\sum\left(w\left(x^{\sigma}\right)\right)-d(0),
$$

for a $w$-invariant $\boldsymbol{Q}$-rational divisor $E>0$ (see (1.11)). Let $\mathscr{L}=\mathcal{O}(D+E)$ $\otimes \mathcal{O}\left(\sum\left(w x^{\sigma}\right)+d(0)\right)^{\otimes(-1)}$ be the invertible sheaf on $\mathscr{X}_{0}(p) \otimes \mathcal{O}_{K}$ for a finite extension $K$ of $\boldsymbol{Q}$. By the upper semicontinuity ([34] (7.7.5), 1), there is a rational function $f$ on $\mathscr{X}_{0}(p) \otimes \bar{F}_{2}$ such that

$$
(f)=2 \sum_{i=1}^{h / 2}\left(y_{i}\right)+E-d(0)-d(\infty)(\neq 0)
$$

for the effective divisor $E=E \otimes F_{2}$ (see (1.10)). The divisor $(f)$ is $\boldsymbol{F}_{2}$-rational and $w$-invariant. Then $w^{*} f=f$ and we may assume that $f$ is defined over $\boldsymbol{F}_{2}$. Then we get a covering $f^{+}$defined over $\boldsymbol{F}_{2}$ :

$$
\mathscr{X}_{0}^{+}(p) \otimes \boldsymbol{F}_{2} \stackrel{f^{+}}{\longrightarrow} \boldsymbol{P}^{1} / \boldsymbol{F}_{2}
$$

such that $\left(f^{+}\right)=\sum_{1=i}^{h / 2}\left(y_{i}\right)+E^{\prime}-d^{\prime}$ (cusp) for an effective divisor $E^{\prime}$ and an integer $d^{\prime}, 1 \leqq d^{\prime} \leqq d$. Here by $y_{i}$ we denote the images of $y_{i}$ by the natural morphism of $\mathscr{X}_{0}(p) \otimes \boldsymbol{F}_{2}$ to $\mathscr{X}_{0}^{+}(p) \otimes \boldsymbol{F}_{2}$, Then $\# \mathscr{X}_{0}^{+}(p)\left(\boldsymbol{F}_{2}\right) \leqq 3+2 d$ $-h / 2$ if $p \equiv 1 \bmod 8 ; \leqq 2+2 d-h / 2$ if $p \equiv 5 \bmod 8$ (see (1.9)).

Case $p \equiv 1 \bmod 8$ and $a=p$. Let $D=D_{1}+D_{2}, D_{1}>\left(x_{1}\right)$, be the decomposition into the sum of $\operatorname{Gal}(H / Q(\sqrt{p}))$-orbits $D_{i}$ of length $h / 2$. Then 


$$
\begin{aligned}
& (g-\sqrt{p})=D_{1}+E_{1}-\sum\left(w\left(x^{\sigma}\right)\right)-d(0) \\
& (g+\sqrt{p})=D_{2}+E_{2}-\sum\left(w\left(x^{\sigma}\right)\right)-d(0),
\end{aligned}
$$

for $\boldsymbol{Q}(\sqrt{p})$-rational, $w$-invariant divisors $E_{i}>0$. Let $1 \neq \sigma$ be an element of the inertia subgroup of a prime of $H$ lying over 2, and $H^{+}=H^{\langle o\rangle}$ the fixed field of $\langle\sigma\rangle(\simeq Z / 2 Z)$. Then $\sigma^{*} D_{i}=D_{i}$ for $i=1,2$. There are only two fixed points of $w$ defined over $H^{+}$(see (1.10)). Therefore $D_{2} \otimes F_{2}=$ $2 \sum^{\prime}\left(y_{i}\right)$. In the same way as above, we get a rational function $f^{+}$on $\mathscr{X}_{0}^{+}(p) \otimes \boldsymbol{F}_{2}$ defined over $\boldsymbol{F}_{2}$ such that $\left(f^{+}\right)=\sum^{\prime}\left(y_{i}\right)+E^{\prime}-d^{\prime}$ (cusp), for an effective divisor $E^{\prime}$ and an integer $d^{\prime}, 1 \leqq d^{\prime} \leqq d$. So $\sharp \mathscr{X}_{0}^{+}(p)\left(F_{2}\right) \leqq$ $2+2 d-h / 4($ see $(1.10))$.

Case $p \equiv 5 \bmod 8$ and $a=p$. Let $D=D_{1}+D_{2}, D_{1}>\left(x_{1}\right)$, be the decomposition into the sum of $\operatorname{Gal}(H / Q(\sqrt{p}))$-orbits $D_{i}$ of length $h / 2$. Here we assume $h=h(-p) \not \equiv 0 \bmod 4$. Then by Lemma (1.11)

$$
\begin{aligned}
& (g-\sqrt{p})=2 D_{1}+E_{1}-\sum\left(w\left(x^{\sigma}\right)\right)-d(0) \\
& (g+\sqrt{p})=2 D_{2}+E_{2}-\sum\left(w\left(x^{\sigma}\right)\right)-d(0),
\end{aligned}
$$

for $Q(\sqrt{ } p)$-rational, $w$-invariant divisors $D_{i}>0$. Let $\sigma=\sigma_{2}$ be the Frobenius element of the rational prime 2. Then $\sigma\left(D_{1}\right)=D_{2}$, i.e., $\left(D_{1} \otimes F_{4}\right)^{(2)}=$ $D_{2} \otimes \boldsymbol{F}_{4} . \quad$ By (1.10), we see that $D_{1} \otimes \boldsymbol{F}_{4}=\left(y_{1}\right)+\sum_{i=2}^{(h-2) / 4}\left(y_{i}\right), \quad y_{1}$ is the $\boldsymbol{F}_{2}$-rational fixed point of $w$ (see (1.10)). By the same way as above, we get a rational function $f^{+}$on $\mathscr{X}_{0}^{+}(p) \otimes \boldsymbol{F}_{4}$ such that $\left(f^{+}\right)=\left(y_{1}\right)+2 \sum_{i=2}^{(h-2) / 4}\left(y_{i}\right)+$ $E^{\prime}-d^{\prime}$ (cusp), for an effective divisor $E^{\prime}$ and an integer $d^{\prime}, 1 \leqq d^{\prime} \leqq d$ (see $(1.10)$ ). Then $\# \mathscr{X}_{0}^{+}(p)\left(\boldsymbol{F}_{4}\right) \leqq 1+4 d-(h-2) / 4$.

Case $p \equiv-1 \bmod 8 . \quad$ Set $D_{1}=\sum_{i=1}^{h}\left(x_{i}\right), D_{2}=\sum_{i=1}^{h}\left(x_{h+i}\right) . \quad$ Then

$$
(g-1)=\left\{\begin{array}{l}
D+E-\sum\left(w\left(x^{\sigma}\right)\right)-d(0) \\
\text { or } \\
2 D_{1}+E_{1}-\sum\left(w\left(x^{\sigma}\right)\right)-d(0)
\end{array}\right.
$$

for $Q$-rational, $w$-invariant divisors $E>0, E_{1}>0$. In both cases, by the same way as above, we get a rational function $f^{+}$on $\mathscr{X}_{0}^{+}(p) \otimes \boldsymbol{F}_{2}$ defined over $F_{2}$ such that $\left(f^{+}\right)=\sum_{i=1}^{h}\left(y_{i}\right)+E^{\prime}-d^{\prime}$ (cusp) for an effective divisor $E^{\prime}$ and an integer $d^{\prime}, 1 \leqq d^{\prime} \leqq d$. Then $\# \mathscr{X}_{0}^{+}(p)\left(F_{2}\right) \leqq 1+2 d-h$ and $\# \mathscr{X}_{0}^{+}(p)\left(F_{4}\right) \leqq 1+4 d-h$ (see $\left.(1.10)\right)$.

Case $p \equiv 3 \bmod 8 . \quad$ Set $D_{1}=\sum_{i=1}^{h}\left(x_{i}\right), D_{2}=\sum_{i=1}^{3 h}\left(x_{i}\right)$. Then

$$
(g-1)=D+E-\sum\left(w\left(x^{\sigma}\right)\right)-d(0)
$$


or

$$
(g+1)=2 D_{2}+E_{2}-\sum\left(w\left(x^{\sigma}\right)\right)-d(0),
$$

for $\boldsymbol{Q}$-rational, $w$-invariant divisors $E>0, E_{2}>0$. In the first case, the same argument as above shows that there is a rational function $f^{+}$on $\mathscr{X}_{0}^{+}(p) \otimes F_{2}$ defined over $F_{2}$ such that $\left(f^{+}\right)=2 \sum_{i=1}^{h}\left(y_{i}\right)+E^{\prime}-d^{\prime}$ (cusp), for an effective divisor $E^{\prime}$ and an integer $d^{\prime}, 1 \leqq d^{\prime} \leqq d$. Then $\# \mathscr{X}_{0}^{+}(p)\left(F_{2}\right) \leqq$ $2+2 d-2 h$, and $\sharp \mathscr{X}_{0}^{+}(p)\left(\boldsymbol{F}_{4}\right) \leqq 1+4 d-h$ (see (1.10)). The second case yields better estimates.

\section{§3. Rational points on $Y_{1}\left(p^{r}\right)$ defined over quadratic fields}

In this section we prove Theorem $\mathrm{A}$ in the introduction. Let $k$ be a quadratic field, $x$ a $k$-rational point on $Y_{1}\left(p^{r}\right)$ for $r \geqq n^{\prime}=n^{\prime \prime}(k, p)$ (see (1.8)). In this case, it is easy to see that $n^{\prime}(k, p)=n^{\prime \prime}(k, p)$ (see (1.7), (1.8)). So we can apply the propositions in Section 2. Moreover, we see that we have only to show $n(k, p)<n^{\prime}(k, p)$ (see Section 0). Applying Proposition (2.3), we get the result of the theorem except for $p=13,37$, $43,67,97,163$ and $193(p<300, \neq 5,7,151,199,227,277)$. See table (4.3).

(3.1). Proof for $p=43,67,73,97,163$ and 193. We can apply (2.4), (2.5) and (2.6) in the last section to these cases. Wada [32] shows that the characteristic polynomials of the Hecke operator $T_{2}$ on the $C$-vector space of holomorphic cusp forms of weight 2 belonging to $\left\langle\Gamma_{0}(p),\left(\begin{array}{cc}0 & -1 \\ p & 0\end{array}\right)\right\rangle$ for $p<250$. According to his table, we get

Table 4 .

\begin{tabular}{rcccc}
\hline \multicolumn{1}{c}{$p$} & characteristic polynomial of $T_{2}$ & $\# \mathscr{X}_{0}^{+}(p)\left(\boldsymbol{F}_{2}\right)$ & $\# \mathscr{X}_{0}^{+}(p)\left(\boldsymbol{F}_{4}\right)$ & $h(-p)$ \\
\hline 43 & $x+2$ & 5 & 5 & 1 \\
67 & $x^{2}+3 x+1$ & 6 & 6 & 1 \\
73 & $x^{2}+3 x+1$ & 6 & 6 & 4 \\
97 & $x^{3}+4 x^{2}+3 x-1$ & 7 & 7 & 4 \\
163 & $x\left(x^{5}+5 x^{4}+3 x^{3}-15 x^{2}-16 x+3\right)$ & 8 & 10 & 1 \\
193 & $\left(x^{2}+3 x+1\right) \times$ & 8 & 12 & 4 \\
\hline
\end{tabular}

With these and Proposition (2.6), we get the proof.

(3.2). Proof for $p=13$. 
(3.2.1). Rational points on $Y_{1}(13)$ defined over quadratic fields $k$.

Let $x$ be a $k$-rational point on $Y_{1}(13)$. There is an elliptic curve $E$ defined over $k$ with a $k$-rational point $P$ of order 13 such that the pair $(E, \pm P$ ) represents $x$ ([2] VI Proposition (3.2)). By (1.5), (1.7), we can apply Lemma (1.9). So there is a rational function $g$ (defined over $\boldsymbol{Q}$ ) on $X_{1}(13)$ such that

$$
(g)=(x)+\left(x^{\sigma}\right)-\left(0_{i}\right)-\left(0_{i_{\sigma}}\right)(\neq 0)
$$

for $1 \neq \sigma \in \operatorname{Gal}(k / Q)$ see (2.1). $g$ defines an involution $\gamma$ of $X_{1}(13)$ such that $X_{1}(13) /\langle\gamma\rangle \simeq \boldsymbol{P}^{1}$. The automorphism [5] $\in \bar{\Gamma}(13)$ (see $\S 1$ ) of $X_{1}(13)$ is of degree 2, and $X_{1}(13) /\langle[5]\rangle \simeq P^{1}$ (see (1.4)). Hence $\gamma=[5]$, and so $x^{\sigma}=$ $\gamma(x), 0_{i_{\sigma}}=\gamma\left(0_{i}\right)\left(\neq 0_{i}\right)$. (Note that if a proper smooth curve $X$ defined over a field is hyperelliptic of genus $\geqq 2$, the involution $\gamma$ satisfying $X \mid\langle\gamma\rangle \simeq \boldsymbol{P}^{1}$ is unique.) Then $\left\{x, x^{\sigma}=\gamma(x)\right\}$ defines a $\boldsymbol{Q}$-rational point on $Y_{1}(13) /\langle\gamma\rangle$ and $0_{i} \otimes F_{q} \neq \gamma\left(0_{i}\right) \otimes F_{q}$ for any rational prime $q$. There exists an elliptic curve $F$ defined over $\boldsymbol{Q}$ such that the image of $G_{\boldsymbol{Q}}=\operatorname{Gal}(\overline{\boldsymbol{Q}} / \boldsymbol{Q})$ of the Galois representation on $F_{13}(\overline{\boldsymbol{Q}})$ is contained in $\left\{\left(\begin{array}{cc}\langle 5\rangle & * \\ 0 & *\end{array}\right)\right\}\left(\subset G L_{2}\left(\boldsymbol{F}_{13}\right)\right)$, and $F \simeq E$ over $C$.

(3.2.2). Suppose that there is a $k$-rational point $x$ on $Y_{1}(169)$. There is an elliptic curve $E$ defined over $k$ with a $k$-rational point $P$ of order $13^{2}$ such that the pair $(E, \pm P$ ) represents $x$ ([2] VI Proposition (3.2)). Let $x^{\prime}$ be a $k$-rational point on $Y_{1}(13)$ which is represented by the pair $\left(E^{\prime}, \pm P^{\prime}\right)_{l k} \overline{\overline{\mathrm{dfn}}}(E /\langle 13 \cdot P\rangle, \pm P \bmod \langle 13 \cdot P\rangle)_{l k}$, and $\rho^{\prime}$ the Galois representation on $E^{\prime}{ }_{13}(\bar{k})$. Then

$$
\rho^{\prime}\left(G_{k}\right) \smile\left\{\left(\begin{array}{ll}
1 & 0 \\
0 & *
\end{array}\right)\right\}
$$

As was seen in (3.2.1), there is an elliptic curve $F$ defined over $\boldsymbol{Q}$ such that the image of $G_{\boldsymbol{Q}}$ under the Galois representation $\rho$ on $F_{13}(\overline{\boldsymbol{Q}})$ is contained in $\left\{\left(\begin{array}{cc}\langle 5\rangle & * \\ 0 & *\end{array}\right)\right\}$ and $E^{\prime} \simeq F$ over $C$. Since $F$ has multiplicative reduction at $q=2$ (see (1.9)), there exists a quadratic extension $K$ of $k$ over which $E^{\prime} \simeq F$. Thus

$$
\rho\left(G_{K}\right) \subset\left\{\left(\begin{array}{ll}
1 & 0 \\
0 & *
\end{array}\right)\right\}
$$

So $\rho\left(G_{Q}\right) \smile\left\{\left(\begin{array}{ll}* & 0 \\ 0 & *\end{array}\right)\right\}$, which contradicts to the fact that $X_{\mathrm{sp} . \mathrm{Car}}(13)(\boldsymbol{Q})$ 
consists of the cusps $0, \infty\left(; X_{\mathrm{sp.Car}}(p) \simeq X_{0}\left(p^{2}\right)\right.$, see [7], [21]).

Remarks (3.3). (3.3.1). The modular curve $X=X_{1}(16)$ is of genus 2 and $\# J_{1}(16)(Q)=20$ (see [6]). Let $X \stackrel{2}{\longrightarrow} Y \stackrel{2}{\longrightarrow} X_{0}(16)$ be the natural covering, $r$ the generator of $\operatorname{Gal}(X / Y)$. Then $Y=X \mid\langle\gamma\rangle \simeq \boldsymbol{P}^{1}$. Let $x$ be a $k$-rational point on $Y_{1}(16)$. If $q=3$ (resp. $=5$ ) does not remain prime in $k$, then $x \otimes \kappa(\mathfrak{q})$ and $x^{\sigma} \otimes \kappa(\mathfrak{q})$ are 0 -cusps for a prime $\mathfrak{q}$ of $k$ lying over $q$ (see (1.9)). Then we get a rational function $g$ on $X$, defined over $\boldsymbol{Q}$, such that $(g)=(x)+\left(x^{\sigma}\right)-\left(0_{i}\right)-\left(0_{i_{\sigma}}\right)$ (see (2.2)). Thus $x^{\sigma}=\gamma(x)$ and $0_{i_{\sigma}}=\gamma\left(0_{i}\right)$ $\left(\neq 0_{i}\right)$ (see (3.2)). Therefore if $q=3$ or 5 ramifies in $k, Y_{1}(16)(k)=\phi$. Let $k$ be an imaginary quadratic field such that the class number of $k$ is prime to 5 and that the rational prime 2 does not split in $k$. Then the fact that $Z / 5 Z \subset J_{1}(16)(Q)$ and the descent ([17] Chapter III) show $\# J_{1}(16)(k)<\infty$. Moreover, if 3 splits in $k$ or 5 does not remain prime in $k$, using Mazur's idea "formal immersion" [18], we see $Y_{1}(16)(k)=\phi$.

(3.3.2). The modular curve $X_{1}(11)$ is an elliptic curve with conductor (11). The defining equation of $X_{1}(11)$ is

$$
y^{2}+y=x^{3}-x^{2}
$$

and $X_{1}(11)(Q) \simeq Z / 5 Z$ (see [35] p. 82). The numbers of the $F_{q^{i}}$-rational points for $q=2,3$ of $\mathscr{X}=\mathscr{X}_{1}(11)$ are as follows:

$$
\begin{array}{ll}
\# \mathscr{X}\left(\boldsymbol{F}_{2}\right)=5, & \# \mathscr{X}\left(\boldsymbol{F}_{4}\right)=5 \\
\# \mathscr{X}\left(\boldsymbol{F}_{3}\right)=5, & \# \mathscr{X}\left(\boldsymbol{F}_{9}\right)=15
\end{array}
$$

(loc. cit.). Therefore $X_{1}(11)(k)_{\text {tor }} \simeq Z / 5 Z$ for quadratic fields $k$. So we have $Y_{1}(11)(k)=\phi$ if and only if the rank of $X_{1}(11)(k)$ is 0 . For example if $k$ is an imaginary quadratic field such that the class number of $k$ is prime to 5 and the rational prime 11 does not split in $k$, then $Y_{1}(11)(k)=\phi$. This can be shown by the descent; see [17] Chapter III.

(3.3.3). By the argument in (3.2.1), we have already known that the $k$-rational points on $Y_{1}(13)$ are parametrized by the $\boldsymbol{Q} \cup\{\infty\}$-values of a rational function on $X_{1}(13) /\langle\gamma\rangle \simeq P_{Q}^{1}$ of degree 1. If the rational prime $q=2$ does not split in $k$ or $q=3$ ramifies in $k$, then $x \otimes \kappa(\mathfrak{q})=x^{\sigma} \otimes \kappa(\mathfrak{q})$ for a $k$-rational point $x$ on $X_{1}(13)$ and a prime $\mathfrak{q}$ of $k$ lying over $q$. Therefore by (3.2.1) in such a case $Y_{1}(13)(k)=\phi$.

(3.4). Proof for $p=37$. Let $X_{1}(37) \stackrel{9}{\longrightarrow} X \stackrel{2}{\longrightarrow} X_{0}(37)$ be the natural coverings, $J=J(X)$ the jacobian variety of $X$ and $A=\operatorname{Coker}\left(J_{0}(37) \rightarrow J\right)$ 
(see (1.4)). Then $A$ has everywhere good reduction over $\boldsymbol{Q}(\sqrt{37})$ ([2] V).

Lemma (3.4.1). Let $p$ be a prime number congruent to $1 \bmod 4$, $X_{1}(p) \stackrel{(p-1) / 4}{\longrightarrow} X \stackrel{2}{\longrightarrow} X_{0}(p)$ the natural coverings, and $J=J(X)$ the jacobian variety of $X$. If there is a prime factor of $(1 / 4) B_{2,(\underline{p})}$ which is prime to the class number of $\boldsymbol{Q}(\sqrt{p})$, then there is a factor $(\mid \boldsymbol{Q}(\sqrt{p}))$ of $\operatorname{Coker}\left(J_{0}(p) \rightarrow J\right)$ with finite Mordell-Weil group $(\mid \boldsymbol{Q}(\sqrt{p}))$. Here $B_{2,(\underline{p})}$ is the (second) generalized Bernoulli number associated to the quadratic residue symbol ( $(\underline{p})$ (see [13]).

Proof. Let $0^{\prime}, 0^{\prime \prime}$ be the 0 -cusps of $X$. The order of $\operatorname{cl}\left(\left(0^{\prime}\right)-\left(0^{\prime \prime}\right)\right)$ is $(1 / 4) B_{2,(\underline{p})}$ [11]. Let $q$ be a prime number which is prime to the class number of $\boldsymbol{Q}(\sqrt{p})$ and divides $(1 / 4) B_{2,(\underline{p})}$. Let $B$ be a quotient ( $\left./ \boldsymbol{Q}\right)$ of Coker $\left(J_{0}(p) \rightarrow J\right)$ such that $B$ is $Q$-simple and the order of the image $\operatorname{cl}\left(\left(0^{\prime}\right)-\left(0^{\prime \prime}\right)\right)$ on $B$ is divisible by $q$, then $Z / q Z \subset B$. $B$ has everywhere good reduction over $Q(\sqrt{p})$, see [2] V, and is isogenous to a product $C \times C^{\sigma}$ of an abelian variety $C$ over $Q(\sqrt{p})$. Further $C$ is isogenous over $\boldsymbol{Q}(\sqrt{p})$ to $C^{\sigma}$ for $1 \neq \sigma \in \operatorname{Gal}(\boldsymbol{Q}(\sqrt{p}) / \boldsymbol{Q})$, see [31] Chapter 7. Then $B$ is isogenous over $\boldsymbol{Q}$ to $\operatorname{Re}_{\boldsymbol{Q}(\sqrt{p}) / \boldsymbol{Q}}(C)$, where $\operatorname{Re}_{\boldsymbol{Q}(\sqrt{p}) / \boldsymbol{Q}}$ is the restriction of scalars (see [4], [33]). Hence $\operatorname{rk} B(\boldsymbol{Q})=\operatorname{rk} C(\boldsymbol{Q}(\sqrt{p})$ ). Applying the descent to $C(\mid \boldsymbol{Q}(\sqrt{p}))$ (see [17] Chapter III), we have $\# C(Q(\sqrt{p}))<\infty$.

Lemma (3.4.2). Let $A=\operatorname{Coker}\left(J_{0}(37) \rightarrow J\right)$ as above. Then $A(\boldsymbol{Q}) \simeq$ $Z / 5 Z$.

Proof. $(1 / 4) B_{2,\left({ }^{37}\right)}=5$ and the class number of $\boldsymbol{Q}(\sqrt{37})=1 . \quad A$ is isogenous over $\boldsymbol{Q}(\sqrt{37})$ to a product of two elliptic curves, so that $A$ is $\boldsymbol{Q}$-simple. Using the table of the characteristic polynomials of the Hecke operators on the $C$-vector space $S_{2}\left(\Gamma_{0}(37),\left({ }^{37}\right)\right)$ of the holomorphic cusp forms of weight 2 with the neben character $\left.{ }^{(37}\right)$ belonging to $\Gamma_{0}(37), \mathrm{p}$. 207 of [31], we see that $\# A(Q)_{\text {tor }}=5$. Then Lemma (3.4.1) is applied to yield $A(\boldsymbol{Q}) \simeq Z / 5 Z$.

Suppose that there is a $k$-rational point $x$ on $Y_{1}(37)$. Consider the $Q$-rational section $i(x)=c 1\left((x)+\left(x^{\sigma}\right)-\left(0_{i}\right)-\left(0_{i_{\sigma}}\right)\right)$ of $A$, where $1 \neq \sigma \epsilon$ $\operatorname{Gal}(k / Q)$, see Section 2. Then $i(x) \otimes F_{q}=0$ for $q=2,3$ and 5 (see (1.9)), so we get $i(x)=0$, see (3.4.2). There is a rational function $g$ on $X$ (defined over $\boldsymbol{Q})$ such that $(g)=(x)+\left(x^{\sigma}\right)+\left(\gamma\left(0_{i}\right)\right)+\left(\gamma\left(0_{i_{\sigma}}\right)\right)-(\gamma(x))-$ $\left(\gamma\left(x^{\sigma}\right)\right)-\left(0_{i}\right)-\left(0_{i_{\sigma}}\right)$, where $1 \neq \sigma \in \operatorname{Aut}\left(X / X_{0}(37)\right)$, see $\left(2.1^{\prime}\right)$. 
Claim. $\quad x \neq \gamma(x), \neq \gamma\left(x^{\sigma}\right)$.

Proof. If $x=\gamma(x)$, then $x$ is a fixed point of $\gamma$ with the modular invariant $j(x)=1728$. This contradicts that $x \otimes \kappa(\mathfrak{q})=0_{i} \otimes \kappa(\mathfrak{q})$ for the primes $\mathfrak{q} \mid 2$ of $k$. If $x=\gamma\left(x^{\sigma}\right)$, then $\left\{x, x^{\sigma}=\gamma(x)\right\}$ defines a $Q$-rational point on $\left(Y_{0}(37)\right)$. But we know that the non-cuspidal $Q$-rational points on $X_{0}(37)$ have everywhere potentially good reduction, [19] Section 5, p. 32 .

Let $\mathscr{X}$ be the normalization of the projective $j$-line $\mathscr{X}_{0}(1) \simeq \boldsymbol{P}_{Z}^{1}$ in $X$. Then $\mathscr{X}$ is smooth over $Z[1 / 37]$, see [2].

Case $0_{i} \neq 0_{i_{\sigma}}$. In this case $\gamma\left(0_{i}\right)=0_{i}$ and $(g)=(x)+\left(x^{\sigma}\right)-(\gamma(x))-$ $\left(\gamma\left(x^{\sigma}\right)\right)(\neq 0)$. Let $E_{\eta}=(x)+\left(x^{\sigma}\right)$ and $E$ be the flat closure of $E_{\eta}$ on $\mathscr{X} \otimes \boldsymbol{Z}_{2}$. Then $E \otimes \boldsymbol{F}_{2}=\left(0_{i} \otimes \boldsymbol{F}_{2}\right)+\left(\gamma\left(0_{i} \otimes \boldsymbol{F}_{2}\right)\right)$. The argument similar to Lemma (1.11) shows that there is a rational function on $\mathscr{X}_{0}(37) \otimes \boldsymbol{F}_{2}$ of degree one. This is a contradiction.

Case $0_{i}=0_{i_{\sigma}}$. Let $E_{\eta}=(x)+\left(x^{o}\right)+2\left(\gamma\left(0_{i}\right)\right)$ and $E$ be the flat closure of $E_{\eta}$ on $\mathscr{X} \otimes Z_{2}$. Then $E \otimes F_{2}=2\left(0_{i} \otimes F_{2}\right)+2\left(\gamma\left(0_{i} \otimes F_{2}\right)\right)$. The argument as in Lemma (1.11) shows that there is a double covering $g^{\prime}: \mathscr{X}_{0}(37) \otimes \boldsymbol{F}_{2} \rightarrow$ $P_{F_{2}}^{1}$, such that $\left(g^{\prime}\right)_{\infty}=2\left(0 \otimes F_{2}\right)$. Then $0=0 \otimes F_{2}$ is a fixed point of the (unique) hyperelliptic involution $\bar{S}$ of $\mathscr{X}_{0}(37) \otimes F_{2}$. The hyperelliptic involution $S$ of $X_{0}(37)$ sends the cusp $0=\left(\begin{array}{l}0 \\ 1\end{array}\right)$ to a non cuspidal $\boldsymbol{Q}$-rational point, see [19] Section 5. As noted as before, $S(0) \otimes F_{2}$ is not a cusp (see loc. cit.), so that $\bar{S}=S \otimes F_{2}$ does not fix $0=0 \otimes F_{2}$. Thus we get a contradiction.

For an imaginary quadratic field $k, Y_{1}(p)(k)=\phi$ if a rational prims $p$ remains prime in $k$, except for finitely many $p$ ([18] $\S 4)$. For a real quadratic field $k$, we use Mazur's idea "formal immersion" (loc. cit.) to show the following.

Proposition (3.5). Let $p \geqq 17$ be a rational prime congruent to $1 \bmod 4$. If there exists a prime factor of $(1 / 4) B_{2,(\underline{p})}$ which is prime to the class number of $\boldsymbol{Q}(\sqrt{p})$, then $Y_{1}(p)(\boldsymbol{Q}(\sqrt{p}))=\phi$.

Proof. Let $X_{1}(p) \stackrel{(p-1) / 4}{\longrightarrow} X \stackrel{2}{\longrightarrow} X_{0}(p)$ be the natural coverings, $J=$ $J(X)$ the jacobian variety of $X$, and $A=\operatorname{Coker}\left(J_{0}(p) \rightarrow J\right)$. Then by Lemma (3.4.1), there exists a quotient $B(/ \boldsymbol{Q})$ of $A$ with finite Mordell-Weil group over $\boldsymbol{Q}(\sqrt{p})$. As $p>(1+3)^{2}$, Lemma (1.8) is applied for $q=3$. The rest owes to [18] Section 4 . 
Corollary (3.6). Let $p$ be a prime number congruent to $1 \bmod 8$. Then $Y_{1}(p)(Q(\sqrt{p}))=\phi$.

Proof. (1/4) $B_{2,(\underline{p})} \equiv 0 \bmod 2$ (see, e.g. [17] Chapter II $\left.\S 12\right)$.

\section{§4. Further results}

Let $k$ be an algebraic number field of degree $d, n=n(k, p)$ and $n^{\prime \prime}=n^{\prime \prime}(k, p)$ as in Section 1 (1.8). Applying propositions in Section 2, we can estimate $n$ in some cases.

Theorem (4.1). Let $k$ be any cubic field. Then

$$
\begin{aligned}
& n(k, 2) \leqq 5 \\
& n(k, 3)=2, \\
& n(k, 17) \leqq 1
\end{aligned}
$$

For $p=19,23,41,47,59,71$ and the primes $p \leqq 79, \neq 97, \neq 109$, satisfying $\# J_{0}^{-}(p)(\boldsymbol{Q})<\infty$, we have $n(k, p)=0$.

Proof. For $p<300$, the result follows from Proposition (2.3), (1.4), (1.8), Lemma (1.12), except for $p=19,23,157,163,193$, (277) (see table (4.3)). Using Corollary (2.5), we get the result for $p=157,163,193$ (see (3.1)). The characteristic polynomial of the Hecke operator $T_{2}$ on $S_{2}\left(\left\langle\Gamma_{0}(157),\left[\begin{array}{cr}0 & -1 \\ 157 & 0\end{array}\right]\right\rangle\right)$ (see (3.1)) is $x^{5}+5 x^{4}+5 x^{3}-6 x^{2}-7 x+1$ (see [32]). Thus $\# \mathscr{X}_{0}^{+}(157)\left(\boldsymbol{F}_{2}\right)=8$ and $\# \mathscr{X}_{0}^{+}(157)\left(\boldsymbol{F}_{4}\right)=10$. For $p=19,23$, if there exists a $k$-rational point $x$ on $Y_{1}(p)$, then there exists a rational function $g$ on $X=X_{1}(p)$, defined over $\boldsymbol{Q}$, such that $(g)=\sum\left(x^{\sigma}\right)-\sum\left(0_{i_{\sigma}}\right)$, see (1.9), (2.1)). For $p=23$, we know $\# \mathscr{X}_{1}(23)\left(F_{2}\right)=11$ ([9] §4). Using the upper semicontinuity (see [34] (7.7.1)1), we get a contradiction.

(4.1.1) Proof for $p=19$. Let $1 \neq \gamma \in \operatorname{Aut}(X / Y)$ (see (1.4)). If $\left(\gamma^{*} g\right)=(g)$, then $\gamma(x)=x^{\tau}$ for a $\tau \in \operatorname{Isom}_{Q}(k, \bar{Q})$. Then $x$ is a fixed point if $\tau=1$, or $\left\{x^{\sigma}\right\}_{\sigma}=\left\{\gamma^{i}(x)\right\}_{i=0,1,2}$ if $\tau \neq 1$. The fixed points of $\gamma$ have the modular invariant $j=0$ (see (1.1), (1.4)). So by Lemma (1.9) the first case above does not occur. In the second case, $\left\{x^{\circ}\right\}_{\sigma}$ defines a $\boldsymbol{Q}$-rational point on $Y$, hence on $X_{0}(19)$. But the $Q$-rational points on $X_{0}(19)$ are the cusps and the points represented by the elliptic curve $C / Z[(1+\sqrt{-19}) / 2]$. So $\left(\gamma^{*} g\right) \neq(g)$ is shown. Let $D=\sum_{i=1}^{6}\left(x_{i}\right)$ be the $\boldsymbol{Q}$-rational divisor of $X_{1}(19)$, where $x_{i}$ are the fixed points of $\gamma$ on $X_{1}(19)$ (see (1.4)). Then by Lemma (1.11), $1 /\left(\gamma^{*} g / g-1\right) \in H^{\circ}\left(X_{1}(19), \mathcal{O}(D)\right)$. The Riemann-Roch theorem and a 
theorem of Clifford (see below) then show $\operatorname{dim}_{Q} H^{0}\left(X_{1}(19), \mathcal{O}(D)\right)<1+3$.

(4.1.2) Theorem (Clifford; see e.g. [5]). Let $X$ be a proper smooth curve $/ C$ of genus $\geqq 1, E$ an effective divisor such that $\operatorname{dim}_{C} H^{0}(X, \mathcal{O}(K-E))>0$, where $K$ is the canonical divisor. Then

$$
\operatorname{dim}_{C} H^{0}(X, \mathcal{O}(E)) \leqq 1+\frac{1}{2} \operatorname{deg}(E)
$$

The equality holds if and only if $E=0, E \sim K$ or $E \sim \pi^{*} F$ if $X$ is hyperelliptic, where $\pi: X \rightarrow \boldsymbol{P}^{1}$ is a double covering and $F$ is a divisor of $\boldsymbol{P}^{1}$.

It is easy to see the following.

(4.1.3). Let $X$ be a proper smooth curve and $r$ an automorphism of $X$ of degree $m$ ( $\geqq 1$ ) defined over $\boldsymbol{Q}$. Let $E$ be an effective, $\boldsymbol{Q}$-rational divisor of $X$ such that $\gamma^{*} E=E$ and $\gamma^{*}$ acts faithfully on $H^{\circ}(X, \mathcal{O}(E)$ ). Then $\operatorname{dim}_{Q} H^{0}(X, \mathcal{O}(E)) \geqq 1+\varphi(m)$, where $\varphi(m)$ is the Euler number of $m$.

Let $\tilde{\gamma}$ be the generator of $\bar{\Gamma}_{0}(19)=\Gamma_{0}(19) / \pm \Gamma_{1}(19)$ (see (1.4)), which is of order 9. Then $D$ is $\tilde{\gamma}^{*}$-invariant and $H^{0}\left(X_{1}(19), \mathcal{O}(D)\right)^{\left\langle\gamma^{*}\right\rangle}=\boldsymbol{Q} \cdot 1$. If $\operatorname{dim}_{Q} H^{0}\left(X_{1}(19), \mathcal{O}(D)\right) \geqq 2$, then $\tilde{\gamma}^{*}$ acts faithfully on $H^{0}\left(X_{1}(19), \mathcal{O}(D)\right)$ and $\operatorname{dim}_{Q} H^{0}\left(X_{1}(19), \mathcal{O}(D)\right) \geqq 1+\varphi(9)=7$ by (4.1.3). This is a contradiction.

(4.1.4) Proof for $p>300$ (e.g. $p=383,419,429,491$, cf. [17] p. 151]) (and for $p=277$ if $\left.\# J_{0}^{-}(277)(Q)<\infty\right)$. By Corollary (2.5), if $Y_{1}(p)(k) \neq \phi$, then $\# \mathscr{X}_{0}(p)\left(F_{4}\right) \leqq 2(1+4 \cdot 3)-s \leqq 24$. But we know \# $\mathscr{X}_{0}(p)\left(F_{4}\right) \geqq 2+(p+1) / 12$ (see [24] Theorem 3). Hence $Y_{1}(p)(k)=\phi$ for $p>300($, and $\mathrm{p}=277$ ) if $\# J_{0}^{-}(p)<\infty$.

Remark (4.2). The above method used for $(p, d)=(19,3)$ can be applied to some other cases. For example, it gives an alternating proof for $(p, d)=(5,2)$. In this case, under the notation in (4.1.1), (4.1.2) and the Riemann-Roch theorem show $\operatorname{dim}_{Q} H^{0}(X, \mathcal{O}(D)) \leqq 1+2$. But if $\operatorname{dim}_{Q} H^{0}(X, \mathcal{O}(D)) \geqq 2$, then it must be $\geqq 1+4$ by (4.1.3).

(4.3). Table for $p<300$.

Let $k$ be an algebraic number field of degree $d$. For the pairs $(p, d)$ in the following table, we get $n(k, p)<n^{\prime \prime}(k, p)$. See (1.4), (1.8), [32], [35] table 5, pp. 135-141. 
Table 5.

\begin{tabular}{|c|c|c|c|}
\hline$p$ & $d$ & $s(p)$ & $h(-p)($ for $p \geqq 23)$ \\
\hline 2 & 2,3 & 8 & \\
\hline 3 & $2,3,4,5$ & 12 & \\
\hline 5 & 2 & 4 & \\
\hline 7 & 2 & 2 & \\
\hline 11 & 2 & 6 & \\
\hline 13 & 2 & 6 & \\
\hline 17 & 2,3 & 8 & \\
\hline 19 & 2,3 & 8 & \\
\hline 23 & 2,3 & 6 & 3 \\
\hline 29 & 2 & 6 & 6 \\
\hline 31 & 2 & 6 & 3 \\
\hline 37 & 2 & 2 & 2 \\
\hline 41 & 2,3 & 8 & 8 \\
\hline 43 & 2 & 4 & 1 \\
\hline 47 & $2,3,4$ & 10 & 5 \\
\hline 53 & 2 & 6 & 6 \\
\hline 59 & $2,3,4,5$ & 12 & 3 \\
\hline 61 & 2 & 6 & 6 \\
\hline 67 & 2 & 4 & 1 \\
\hline 71 & $2,3,4,5,6$ & 14 & 7 \\
\hline 73 & 2 & 4 & 4 \\
\hline 79 & $2,3,4$ & 10 & 5 \\
\hline 83 & $2,3,4,5$ & 12 & 3 \\
\hline 89 & $2,3,4,5$ & 12 & 12 \\
\hline 97 & 2 & 4 & 4 \\
\hline 101 & $2,3,4,5,6$ & 14 & 14 \\
\hline 103 & $2,3,4$ & 10 & 5 \\
\hline 107 & $2,3,4,5$ & 12 & 3 \\
\hline 109 & 2 & 6 & 6 \\
\hline 113 & 2,3 & 8 & 8 \\
\hline 127 & $2,3,4$ & 10 & 5 \\
\hline 131 & $2,3, \cdots, 9$ & 20 & 5 \\
\hline 137 & 2,3 & 8 & 8 \\
\hline 139 & $2,3,4,5$ & 12 & 3 \\
\hline 149 & $2,3,4,5,6$ & 14 & 14 \\
\hline 151 & $?$ & 14 & 7 \\
\hline 157 & 2,3 & 6 & 6 \\
\hline 163 & 2,3 & 4 & 1 \\
\hline
\end{tabular}


Table 5. Continued

\begin{tabular}{clrc}
\hline$p$ & \multicolumn{1}{c}{$d$} & $s(p)$ & $h(-p)$ (for $p \geqq 23)$ \\
\hline 167 & $2,3, \cdots, 10$ & 22 & 11 \\
173 & $2,3,4,5,6$ & 14 & 14 \\
179 & $2,3, \cdots, 9$ & 20 & 5 \\
181 & $2,3,4$ & 10 & 10 \\
191 & $2,3, \cdots, 12$ & 26 & 13 \\
193 & 2,3 & 4 & 4 \\
197 & $2,3,4$ & 10 & 10 \\
199 & $\quad ?$ & 18 & 9 \\
211 & $2,3,4,5$ & 12 & 3 \\
223 & $2,3,4,5,6$ & 14 & 7 \\
227 & $?$ & 20 & 5 \\
229 & $2,3,4$ & 10 & 10 \\
233 & $2,3,4,5$ & 12 & 12 \\
239 & $2,3, \cdots, 14$ & 30 & 15 \\
241 & $2,3,4,5$ & 12 & 12 \\
251 & $2,3, \cdots, 13$ & 28 & 7 \\
257 & $2,3,4,5,6,7$ & 16 & 16 \\
263 & $2,3, \cdots, 12$ & 26 & 13 \\
269 & $2,3, \cdots, 10$ & 22 & 22 \\
271 & $2,3, \cdots, 10$ & 22 & 11 \\
277 & $\quad ?$ & 6 & 6 \\
281 & $2,3, \cdots, 9$ & 20 & 20 \\
283 & $2,3,4,5$ & 12 & 3 \\
293 & $2,3, \cdots, 8$ & 18 & 18 \\
\hline
\end{tabular}

\section{REFERENCES}

[1] Z. I. Borevich, I. R. Shafarevich, Number theory, Academic Press, New York and London (1966).

[2] P. Deligne, M. Rapoport, Les schémas de modules de courbes elliptiques, Proceedings of the International Summer School on Modular functions of one variable, vol. II, Lecture Notes in Math., 349, Springer-Verlag, Berlin-Heiderberg-New York (1973).

[ 3 ] B. H. Gross, Arithmetic on elliptic curves with complex multiplication, Lecture Notes in Math., 776, Springer-Verlag, Berlin-Heiderberg-New York (1980).

[4] A. Grothendieck, Fondements de la géométrie algébrique, Sém. Bourbaki, 19571962.

[5] R. Hartshorne, Algebraic Geometry, Springer-Verlag, New York (1977).

[6] M. A. Kenku, Certain torsion points on elliptic curves defined over quadratic fields, J. London Math. Soc., (2) 19 (1979), 233-240.

[ 7 ] - The modular curve $X_{0}(169)$ and rational isogeny, J. London Math. Soc., (2) $22(1980), 239-244$. 
[ 8 ] - On the modular curves $X_{0}(125), X_{1}(25)$ and $X_{1}(49)$, J. London Math. Soc., (2) 23 (1981), 415-427.

[9] - Rational torsion points on elliptic curves defined over quadratic fields, to appear.

[10] D. Kubert, Universal bounds on the torsion of elliptic curves, Proc. London Math. Soc., (3) 33 (1976), 193-237.

[11] D. Kubert, S. Lang, Units in the modular function fields I, II, III, Math. Ann., 218 (1975), 67-96, 175-189, 273-285.

[12] S. Lang, Elliptic Functions, Addison-Wesley, Reading Math. (1973).

[13] — Cyclotomic Fields, Addison-Wesley, Reading Math. (1973).

[14] Yu. I. Manin, The $p$-torsion of elliptic curves is uniformly bounded, Math. USSRIzv., 3 (1969), 433-438.

[15] - Parabolic points and zeta functions of modular curves, Math. USSR-Izv., 6 (1972), 19-64.

[16] B. Mazur, Rational points on modular curves, Proceedings of Conference on Modular Functions held in Bonn, Lecture Notes in Math., 601, Springer-Verlag, BerlinHeiderberg-New York (1977).

[17] - Modular curves and the Eisenstein ideal, I.H.E.S. Publ. Math., 47 (1977), $33-186$.

[18] — Rational isogenies of prime degree, Invent. Math., 44 (1978), 129-162.

[19] —, P. Swinnerton-Dyer, Arithmetic of Weil curves, Invent. Math., 25 (1974), $1-61$.

[20] — J. Tate, Points of order 13 on elliptic curves, Invent. Math., 22 (1973), $41-49$.

[21] J. F. Mestre, Points rationnels de la courbe modulaire $X_{0}(169)$, Ann. Inst. Fourier, 30, 2 (1980), 17-27.

[22] A. Ogg, Rational points on certain elliptic modular curves, Proc. Symposia in Pure Math. XXIV, AMS (1973), 221-231.

[23] —- Hyperelliptic modular curves, Bull. Soc. Math. France, 102 (1974), 449-462.

[24] - Diophantine equation and modular forms, Bull. Amer. Math. Soc., 81 (1975), 14-27.

[25] F. Oort, J. Tate, Group schemes of prime order, Ann. Sci. Ecole Norm. Sup. série 4,3 (1970), 1-21.

[26] M. Raynaud, Schémas en groupes de type $(p, \ldots, p)$, Bull. Soc. Math. France, 102 (1974), 241-280.

[27] K. A. Ribet, Endomorphisms of semi-stable abelian varieties over number fields, Ann. of Math., 101 (1975), 555-562.

[28] J. P. Serre, $p$-torsion des courbes elliptiques (d'àpres Y. Manin), Sém. Bourbaki 1969/70 pp. 281-294, Lecture Notes in Math., 180, Springer-Verlag, BerlinHeiderberg-New York (1971).

[29] J. P. Serre, Corps Locaux, Publ. Inst. de Math. de Univ. de Nancago Hermann, Paris (1968).

[30] G. Shimura, On elliptic curves with complex multiplication as factors of jacobians of modular function fields, Nagoya Math. J., 43 (1971), 199-208.

[31] - Introduction to the Arithmetic theory of Automorphic Functions, Publ. Math. Soc. Japan 11, Iwanami Shoten, Tokyo-Princeton Univ. Press, Princeton, N.J.

[32] H. Wada, A table of Hecke operators II, Proc. Japan Acad., 49 (1973) 380-384.

[33] A. Weil, Adèles and Algebraic Groups, Lecture Notes, Inst. for Advanced Study, Princeton, N.J. 
[34] Éléments de Géométrie Algébrique III (par A. Grothendieck), I.H.E.S. Publ. Math., 17 (1963).

[35] Modular Functions of One Variable IV (Ed. By B. J. Birch and W. Kuyk), Lecture Notes in Math., 476, Springer-Verlag, Berlin-Heiderberg-New York (1975).

Department of Mathematics

Faculty of Science

University of Tokyo

Hongo, Tokyo 113

Japan 